Portland State University

PDXScholar

\title{
Overcoming Barriers for the Wide-scale Adoption of Standardized Real-time Transit Information
}

\author{
Sean J. Barbeau \\ University of South Florida
}

Follow this and additional works at: https://pdxscholar.library.pdx.edu/trec_reports

Part of the Transportation Commons, and the Urban Studies Commons Let us know how access to this document benefits you.

\section{Recommended Citation}

Barbeau, Sean J. Overcoming Barriers for the Wide-scale Adoption of Standardized Real-time Transit Information. NITC-RR-1062. Portland, OR: Transportation Research and Education Center (TREC), 2018. https://doi.org/10.15760/trec.199

This Report is brought to you for free and open access. It has been accepted for inclusion in TREC Final Reports by an authorized administrator of PDXScholar. Please contact us if we can make this document more accessible: pdxscholar@pdx.edu. 

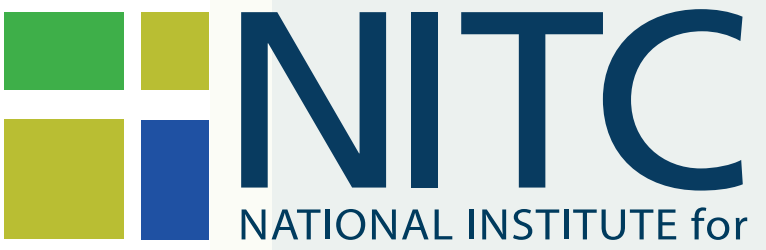

TRANSPORTATION and COMMUNITIES

FINAL REPORT

Overcoming Barriers for the Wide-scale Adoption of Standardized Real-time Transit Information

NITC-RR-1062 a April 2018

NITC is a U.S. Department of Transportation

national university transportation center.

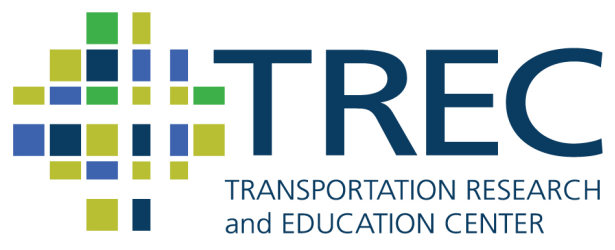





\title{
OVERCOMING BARRIERS FOR THE WIDE-SCALE ADOPTION OF STANDARDIZED REAL-TIME TRANSIT INFORMATION
}

\author{
Final Report \\ NITC-RR-1062 \\ by \\ Dr. Sean J. Barbeau \\ Center for Urban Transportation Research \\ University of South Florida
}

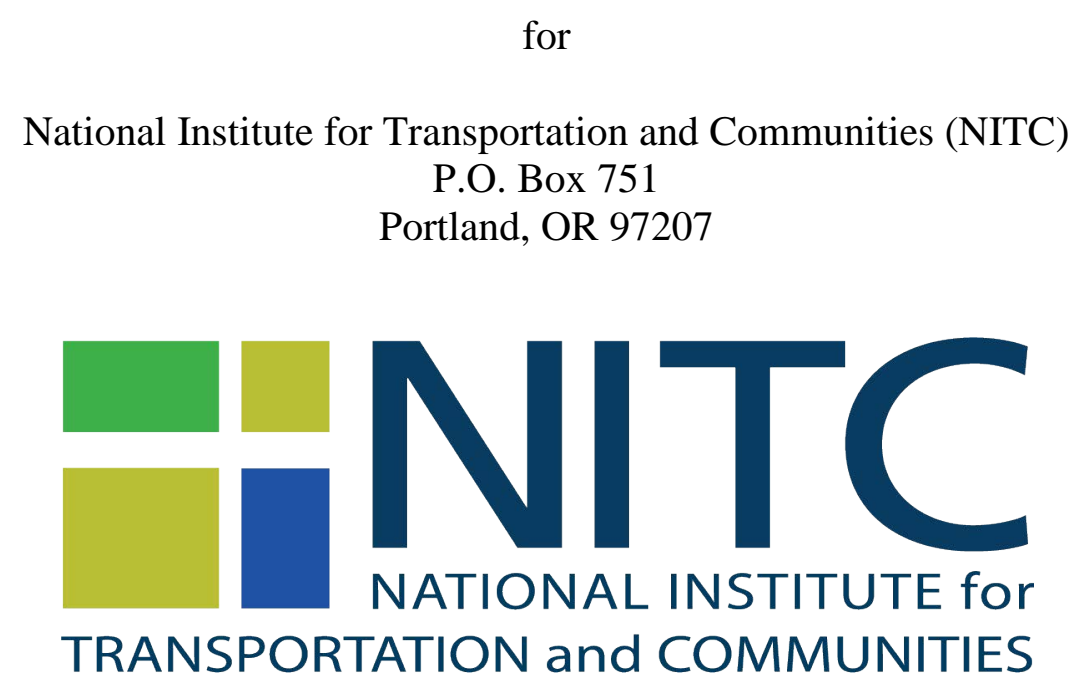

April 2018 


\section{Technical Report Documentation Page}

\begin{tabular}{|l|l|l|}
\hline $\begin{array}{l}\text { 1. Report No. } \\
\text { NITC-RR-1062 }\end{array}$ & 2. Government Accession No. & 3. \\
\hline
\end{tabular}

\section{Title and Subtitle}

Overcoming Barriers for the Wide-scale Adoption of Standardized Real-time Transit Information

7. Author(s)

3. Recipient's Catalog No.
$\begin{aligned} & \text { 5. Report Date } \\ & \text { April } 2018\end{aligned}$

6. Performing Organization Code

8. Performing Organization Report No.

10. Work Unit No. (TRAIS)

9. Performing Organization Name and Address

Center for Urban Transportation Research

University of South Florida

4202 E. Fowler Ave., CUT100

Tampa, FL 33620

12. Sponsoring Agency Name and Address

National Institute for Transportation and Communities (NITC)

P.O. Box 751

Portland, OR 97207

15. Supplementary Notes

16. Abstract

Real-time transit information has many benefits to transit riders and agencies, including shorter perceived and actual wait times, a lower learning curve for new riders, an increased feeling of safety, and increased ridership. In the last few years, a real-time complement to the General Transit Feed Specification (GTFS) format, GTFS-realtime, has emerged. GTFS-realtime has the potential to standardize real-time data feeds and lead to widespread adoption for transit agencies and multimodal apps. However, GTFS-realtime v1.0 has suffered from a lack of clear documentation and openly available validation tools, which significantly increases the time and effort necessary to create and maintain GTFS-realtime feeds. More importantly, bad data have been shown to have a negative effect on ridership, the rider's opinion of the agency, and the rider's satisfaction with multimodal apps.

This project focused on the community-driven creation of the GTFS-realtime v2.0 format, which establishes better guidance for transit agencies, application developers, and automatic vehicle location (AVL) system vendors on what fields are required or optional under various transit use cases. The research team also collaborated with the GTFS community to create GTFS Best Practices. In parallel to these standardization efforts, an open-source GTFS-realtime validation tool (https://github.com/CUTR-at-USF/gtfs-realtime-validator) was developed to allow these same parties to quickly identify and resolve problems in a feed. To demonstrate the utility of the GTFS-realtime Validator and capture the current state of real-time data quality in the industry, the Transit Feed Quality Calculator (https://github.com/CUTR-at-USF/transitfeed-quality-calculator) tool was created to automatically download and validate a large number of agency feeds. An evaluation of 78 transit agency GTFS-realtime feeds showed errors in 54 feeds and warnings in 58 feeds, indicating widespread problems with quality control across many agencies and AVL vendors.

Future work should focus on encouraging agencies to use GTFS-realtime v2.0 and the GTFS-realtime Validator, especially when specifying requirements in RFPs for new AVL systems. A hosted instance of the GTFS-realtime Validator would be useful for agencies that cannot run the tool themselves (e.g., due to internal IT policies preventing installation of applications). Official GTFS-realtime Best Practices voted upon by the GTFS community, similar to GTFS Best Practices, should also be created. Some gray areas remain in the GTFS-realtime specification that should be clarified via future proposals to the GTFS community, and new validator rules based on these clarifications could also be created. A data dashboard that shows the current quality of industry feeds may help agencies and vendors better understand how they relate to their peers in terms of data quality. GTFS may benefit from a more formal governance structure going forward, while being careful not to abandon key qualities of the grassroots approach to governance that has served the format well to date. Finally, the research into how the adoption of GTFS-realtime v2.0 and the GTFS-realtime validator impact data quality over time could be examined, as well as the possible institutional barriers that prevent some agencies from acknowledging and resolving errors in the data.

17. Key Words

Transit, public transportation, real-time information, data, GTFS, GTFS-

18. Distribution Statement

No restrictions. Copies available from NITC: realtime, quality www.nitc.us

19. Security Classification (of this report)

Unclassified
21. No. of Pages

52 



\section{ACKNOWLEDGEMENTS}

This project was funded by the National Institute for Transportation and Communities (NITC) under grant number NITC-RR-1062. The PI would like to acknowledge the Google Summer of Code program, which funded Nipuna Gunathilake's work on a very early version of the opensource GTFS-realtime Validator software tool. Thanks to Mohan Gandhi Achchakkagari and Surya Vamshi Kandukoori for their software development work on the GTFS-realtime Validator during this project, and Surya Vamshi Kandukoori for his contributions to the open-source Transit Feed Quality Calculator tool as well as his efforts in collecting, analyzing, and visualizing errors and warnings found in feeds.

\section{DISCLAIMER}

The contents of this report reflect the views of the authors, who are solely responsible for the facts and the accuracy of the material and information presented herein. This document is disseminated under the sponsorship of the U.S. Department of Transportation University Transportation Centers Program and Florida Department of Transportation in the interest of information exchange. The U.S. Government and Florida Department of Transportation assume no liability for the contents or use thereof. The contents do not necessarily reflect the official views of the U.S. Government and Florida Department of Transportation. This report does not constitute a standard, specification, or regulation.

\section{RECOMMENDED CITATION}

Barbeau, Sean J. Overcoming Barriers for the Wide-scale Adoption of Standardized Real-time Transit Information. NITC-RR-1062. Portland, OR: Transportation Research and Education Center (TREC), 2017. 



\section{TABLE OF CONTENTS}

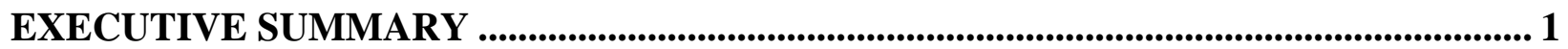

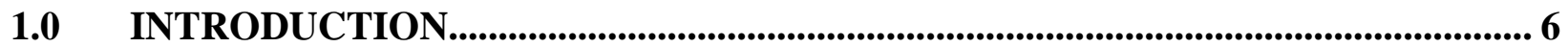

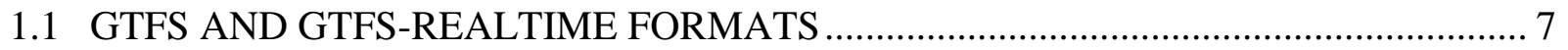

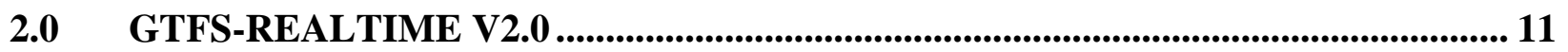

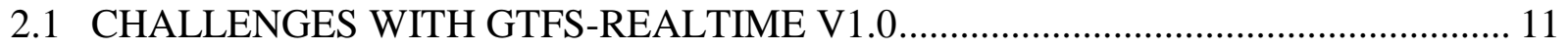

2.2 DEFINING TRANSIT-SPECIFIC FIELD REQUIREMENTS …................................ 16

IMPROVING GTFS DATA UNIFORMITY ......................................................... 19

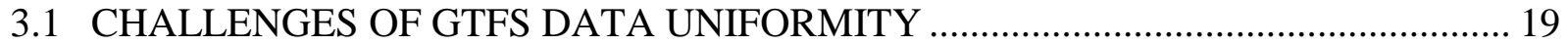

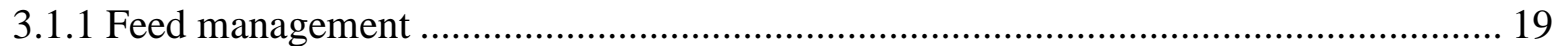

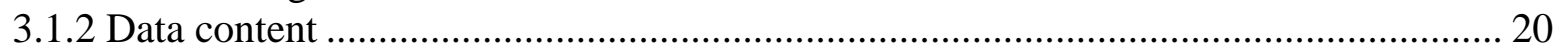

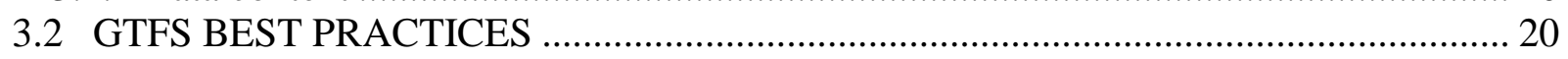

3.3 ADDITIONAL GTFS AND GTFS-REALTIME IMPROVEMENTS ......................... 22

4.0 GTFS-REALTIME VALIDATION TOOL ............................................................ 23

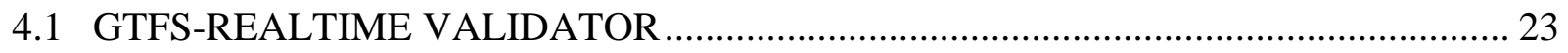

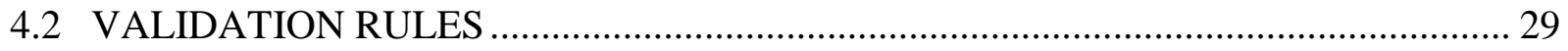

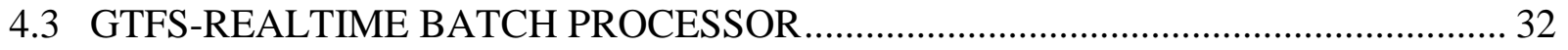

5.0 ANALYSIS OF GTFS-REALTIME FEEDS ......................................................... 33

6.0 CONCLUSIONS AND FUTURE WORK .............................................................. 37

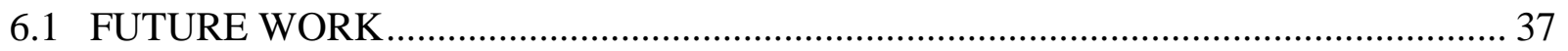

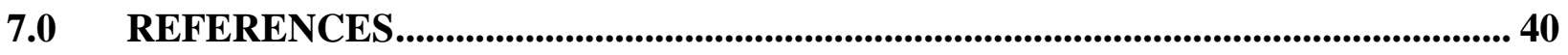




\section{LIST OF TABLES}

Table 1.1 - An example of data from the GTFS format stop_times.txt file................................ 8

Table 1.2 - An example of data from a GTFS-realtime Trip Update message ............................ 9

Table 2.1 - A fully compliant GTFS-realtime v1.0 Vehicle Position ......................................... 11

Table 2.2 - A missing stop_sequence value is problematic for routes with loops...................... 12

Table 2.3 - An excerpt of the gtfs-realtime.proto file ...................................................... 14

Table 4.1 - Errors currently implemented in the GTFS-realtime Validator ............................... 29

Table 4.2 - Warnings currently implemented in the GTFS-realtime Validator ............................ 30

Table 4.3 - Example output from the Batch Processor validation tool ........................................ 32 


\section{LIST OF FIGURES}

Figure 1 - Typical real-time information flow from a transit agency to a mobile app.......................................10

Figure 2 - A route that includes a loop that visits stop_id A more than once ..................................................... 12

Figure 3 - An example of the space savings of the binary Protocol Buffer format..........................................13

Figure 4 - The Protocol Buffer compiler autogenerates code to exchange binary GTFS-realtime messages.....15

Figure 5 - Example of GTFS-realtime v2.0 showing new "Required" and "Cardinality” fields .........................17

Figure 6 - The stop_sequence field as defined in GTFS-realtime v2.0............................................................18

Figure 7 - The new GTFS Best Practices, available at http://gtfs.org/best-practices/......................................21

Figure 8 - GTFS-realtime Validator tool source code on GitHub......................................................................23

Figure 9 - The first screen of the GTFS-realtime Validator............................................................................24

Figure 10 - The monitoring screen, with a summary of all errors and a log of each feed iteration ....................25

Figure 11 - Error and warning IDs (e.g., E002) shown next to every record in the Summary and Log sections26

Figure 12 - Two rules from the validator rule documentation on GitHub ........................................................27

Figure 13 - The validator screen showing the ID of the last iteration and the time when it was validated ........27

Figure 14 - The Iteration Details screen shows the GTFS-realtime message (left) and occurrences of each error

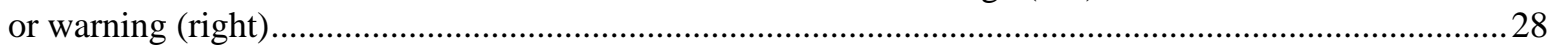

Figure 15 - All errors and warnings for a feed message can be downloaded in CSV format ...........................29

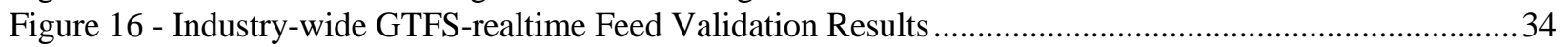

Figure 17 - Most Frequent Errors and Warnings in GTFS-realtime Feeds.......................................................34

Figure 18 - Distribution of Error Frequencies in GTFS-realtime Feeds .........................................................35 



\section{EXECUTIVE SUMMARY}

Real-time transit information has been shown to have many benefits to transit riders, including shorter perceived wait time [1], shorter actual wait time [1], a lowered learning curve for new riders [2], and increased feeling of safety (e.g., at night) [3, 4]. Transit agencies that have deployed real-time information have also benefitted from increased ridership [5, 6], as well as a better perception of the agency and its transit service, even if its service hasn't actually changed [7].

Availability of transit schedule, stop, and route information to transit riders via mobile apps has historically been driven by agencies sharing this data in the General Transit Feed Specification (GTFS) format [8], which has become the dominant format for open schedule data in the transit industry and shared by over 1,500 agencies worldwide [9].

In the last few years, a real-time counterpart to GTFS, GTFS-realtime [10], has begun to emerge, with agencies sharing their real-time data in this format. Previously, real-time transit information had only been shared in proprietary formats specific to each vendor or agency. GTFS-realtime offers the opportunity for application developers to create a mobile app that can function across a large number of cities and agencies, and for practitioners and researchers to be able to easily study and compare actual system performance across different transit systems using the same tools, without the overhead of manually transforming data into a consistent format. Having real-time transit data available in a common format is a key pillar for real-time multimodal information systems.

Data quality is of equal importance to data availability. In fact, accuracy of real-time information is a key concern of transit riders. A survey of riders of a mobile transit app showed that $84 \%$ rely solely on real-time information instead of using the schedule [4]. Errors in predictions create a negative perception of the mobile app providing the information as well as the transit agency. For example, 74\% of surveyed Puget Sound transit riders considered a difference between actual and estimated arrival times greater than four minutes as an "error.” In addition, $9 \%$ of surveyed riders said that they took the bus less often due to errors they experienced [4]. Prediction errors can also lead to reduced system performance if transit agency operations personnel are making decisions based on this data.

This project focused on the collaborative creation of the GTFS-realtime v2.0 format with the GTFS community, which establishes better guidance for transit agencies, application developers, and automatic vehicle location system vendors on what fields are required or optional under various transit use cases. 
GTFS-realtime uses Protocol Buffers, a binary format that compresses data, to exchange information between app developers and transit agencies. In GTFS-realtime v1.0, transit data field requirements were copied from the Protocol Buffer configuration file. Protocol Buffer configurations typically define most fields as "optional” for better forward compatibility (i.e., so software can be changed without breaking communication with older versions). However, this documentation error resulted in labeling many transit data fields as "Optional" when they should have been required under certain transit use cases. AVL vendors used this documentation when creating feeds, resulting in some data being erroneously omitted. GTFS-realtime v2.0 defines new properties for each field, including a new "Required" field that can have the values of Required, Conditionally Required, or Optional depending on the transit use case. Fields that are Conditionally Required have information in the Description property that defines when they are optional and when they are required. An example of a Conditionally Required field is that stop_sequence is now required within a trip_update if that trip has a loop that visits a stop more than once.

GTFS differs from other data standards in that it is largely a grassroots effort driven by transit agencies that produce the data as well as application developers who consume it in their applications - there is no official balloted standards organization (e.g., APTA, IEEE, ISO) that controls the format. GTFS has a community-driven process to adopt changes to the format, where anyone can propose a change (e.g., the changes for GTFS-realtime v2.0 created by the research team) that is then voted on by others in the community. However, there is a high bar for adoption of changes - a change must be implemented by both an agency as well as an application developer before a vote can be opened. Additionally, for a change to be adopted the vote must have unanimous approval - any votes against the proposal will prevent it from being adopted.

While the above governance model has served GTFS well in keeping it focused on a core set of services surrounding customer-facing trip planning and real-time information systems, it has resulted in some useful ideas that are endorsed by the majority, but not all, of those using GTFS being left out of the GTFS documentation. The research team collaborated with some of the most active members in the GTFS community [11] to create the GTFS Best Practices (http://gtfs.org/best-practices/) that are based on these concepts. These best practices help address some of the major challenges in data fragmentation and provide guidance to transit agencies, vendors, and consultants helping to produce and consume GTFS data, as well as app developers. GTFS Best Practices differ from the GTFS reference specification in that Best Practices only require a majority vote for adoption, while changes to the GTFS reference specification require unanimous consent. Additionally, GTFS Best Practices include guidance on how a feed should be managed and updated, which is beyond the scope of the GTFS reference specification that only governs the data format. 
In parallel to these standardization efforts, an open-source GTFS-realtime validation tool (https://github.com/CUTR-at-USF/gtfs-realtime-validator) was developed to allow transit agencies, AVL vendors, and application developers to quickly identify and resolve problems in a GTFS-realtime feed. The GTFS-realtime Validator developed under this project was designed as an open-source tool to be downloaded and run on any computer. It includes a web application interface were users can enter a GTFS and GTFS-realtime URL and instantly get feedback on detected errors in the feed, including links to view the GTFS-realtime message that contains the error as well as the precision location in the feed where the error is contained. Users can share reports with others simply by copying and sharing the web application URL, or by clicking on a button to download the results to a comma-separated value (CSV) format. The GTFS-realtime Validator rules are implemented as a separate module within the project, which allows other developers to integrate these rules into their own application. A Batch Processor tool is also included within the library, which can use downloaded GTFS and GTFS-realtime data as input and then generate the validation results as output in the JavaScript Object Notation (JSON) format. These features allow other applications to integrate GTFS-realtime validation easily into their own systems.

To demonstrate the utility of the GTFS-realtime Validator and capture the current state of realtime data quality in the industry, the Transit Feed Quality Calculator (https://github.com/CUTRat-USF/transit-feed-quality-calculator) tool was created to automatically download and validate many agency feeds. This tool retrieves a list of known GTFS-realtime feed URLs from TransitFeeds.com as well as a comma-separated value (CSV) file and downloads GTFS and GTFS-realtime data from each of these URLs. It then runs the GTFS-realtime Validator Batch Processor on all these feeds to generate an Excel file and JSON output with a summary of errors and warnings found in all of the analyzed feeds. An evaluation of 78 publicly available transit agency GTFS-realtime feeds (feeds without access restrictions listed in the feed directory TransitFeeds.com) showed integrity errors (e.g., trip IDs not matching GTFS, arrival predictions for stops that are out of order), in 54 feeds and warnings in 58 feeds, indicating widespread problems with quality control across many agencies and automatic vehicle location (AVL) vendors.

Now that the GTFS community has accepted GTFS-realtime v2.0, future work should focus on encouraging transit agencies to adopt it, especially when they create Requests for Proposals (RFPs) for new AVL systems. Possible strategies to increase awareness include announcements by government and industry organizations such as the Federal Transit Administration (FTA) and the American Public Transportation Association (APTA), as well as state departments of transportation and statewide public transportation organizations (e.g., FPTA). The research team created a presentation for GTFS-realtime v2.0 for a TransitCenter Transit Data Workshop [12] as well as a paper and poster for the 2018 Transportation Research Board conference [13] and a short blog post [14] announcing GTFS-realtime v2.0. Future conference presentations and webinars can utilize these same materials. Given that the U.S. Department of Transportation has 
already requested GTFS data from agencies as part of the National Transit Map initiative [15], transit agencies may benefit from similar guidance regarding GTFS-realtime emerging as a de facto standard.

The research team has also identified additional issues that require clarification in the GTFS and GTFS-realtime specification [16]. Additional new rules (identified with the "new rule" label [17]) could also be added to the validator, including a few that first require proposals to clarify a portion of the GTFS-realtime documentation. Several transit agencies voiced the desire to have a hosted instance of the GTFS-realtime Validator tool instead of running it themselves. Future work should focus on establishing a long-term server and support where the GTFS-realtime Validator tool can be hosted [18].

Discussions with some transit agencies also uncovered a lack of knowledge for what is currently possible with GTFS-realtime. For example, some agencies were not aware that trips can be canceled using the current GTFS-realtime specification. Future work could focus on creating better documentation in layman's terms that identifies these common use cases and clearly outlines what is and is not possible given the current specification. Future work could also focus on creating official GTFS-realtime Best Practices documentation via the GTFS community process, similar to how the GTFS Best Practices were established. For example, many of the warnings created for the GTFS-realtime validator are likely to be considered best and/or standard practices by the majority of the community and could be officially adopted via a vote.

The Transit Feed Quality Calculator tool can be improved - future work could focus on compiling additional feeds not currently documented in TransitFeeds.com, as well as utilizing a CSV file to store some URLs for feeds that require API keys [19]. Several agencies and application developers have expressed interest in a real-time "Data Dashboard" for GTFSrealtime feeds that would show the number of errors and warnings for all feeds for all agencies, as identified by the GTFS-realtime Validator. This dashboard may help an agency better understand how it is currently performing in real-time data quality, and how they compare to their peers. This same infrastructure can also collect and analyze data to drive evidence-based standard practices.

While the GTFS community has benefitted from the grassroots approach to governance in the past, given the substantial number of stakeholders for both GTFS and GTFS-realtime data the community may benefit from a more formal, organized structure going forward. The GTFS Best Practices effort showcases what can be accomplished relatively quickly by a smaller subset of organizations focused on accomplishing a specific goal, and a similar strategy will likely be required for creating GTFS-realtime Best Practices. This emerging coordination effort should be careful not to lose sight of the benefits of the grassroots approach that has made GTFS a widely adopted format (e.g., requiring producers and consumers for every new proposal to discourage speculative additions to the format). A more formal organization facilitating this coordination 
could also host useful resources and tools for producers and consumers of the data (e.g., the hosted GTFS-realtime Validator, the “Data Dashboard”).

Finally, there is research to be performed in the area of transit agency management and supporting information technology related to institutional barriers of acknowledging and resolving data problems. In the research team's experience, even if a data error is reported to an agency it is not always fixed. Additionally, some transit agencies fix data problems after they are reported much more quickly than others. Identifying the successful practices that triage and resolve data issues quickly may help other agencies that struggle with this process. 


\subsection{INTRODUCTION}

Real-time transit information has been shown to have many benefits to transit riders, including shorter perceived wait time [1], shorter actual wait time [1], a lowered learning curve for new riders [2], and increased feeling of safety (e.g., at night) [3, 4]. Transit agencies that have deployed real-time information have also benefitted from increased ridership [5, 6], as well as a better perception of the agency and its transit service, even if its service hasn't actually changed [7].

Availability of transit schedule, stop, and route information to transit riders via mobile apps has historically been driven by agencies sharing this data in the GTFS format [8], which has become the dominant format for open schedule data in the transit industry and shared by over 1,500 agencies worldwide [9]. In the last few years, a real-time counterpart to GTFS, GTFS-realtime [10], has begun to emerge, with agencies sharing their real-time data in this format. Previously, real-time transit information had only been shared in proprietary formats specific to each vendor or agency. GTFS-realtime offers the opportunity for application developers to create a mobile app that can function across a large number of cities and agencies, and for practitioners and researchers to be able to easily study and compare actual system performance across different transit systems using the same tools, without the overhead of manually transforming data into a consistent format. Having real-time transit data available in a common format is a key pillar for real-time multimodal information systems.

However, of equal importance to data availability is data quality. In fact, accuracy of real-time information is a key concern of transit riders. A survey of riders of a mobile transit app showed that $84 \%$ rely solely on real-time information instead of using the schedule [4]. Errors in predictions create a negative perception of the mobile app providing the information as well as the transit agency. For example, 74\% of surveyed Puget Sound transit riders considered a difference between actual and estimated arrival times greater than four minutes as an "error." In addition, 9\% of surveyed riders said that they took the bus less often due to errors they experienced [4]. Prediction errors can also lead to reduced system performance if transit agency operations personnel are making decisions based on this data.

The following sections of this chapter present an introduction to the GTFS and GTFS-realtime formats. Subsequent chapters discuss how GTFS-realtime v2.0 and a new GTFS-realtime Validation tool can help agencies improve the quality of their real-time data. 


\subsection{GTFS AND GTFS-REALTIME FORMATS}

GTFS forms the foundation for a GTFS-realtime feed - a GTFS-realtime feed cannot provide practical real-time prediction information without having a companion GTFS feed that defines the schedule. GTFS data is implemented as a set of comma-delimited text files added to a single zip file [8].

A subset of the full GTFS specification is required for a GTFS-realtime feed - the following are key for understanding real-time information:

- $\quad$ stops.txt - All bus stops included in a feed, with each record including a stop_id (identifier internal to agency), stop_code (rider-facing stop identifier), stop location, location_type (a single stop or station with multiple stops), etc. For some agencies, stop_id and stop_code may be the same.

- $\quad$ routes.txt - All routes defined for an agency, including a route_id and short and long name.

- calendar.txt and calendar_dates.txt - Includes service days and times, each identified via a service_id, that the agency provides service.

- trip.txt - All trips defined for an agency, including to which route_id each trip belongs. A route may have multiple trip patterns, depending on the day and/or time. The day/time that each trip is operational is defined by a service_id that relates to calendar.txt and/or calendar_dates.txt.

- stop_times.txt - The core schedule file that defines, for each trip_id, the ordered list of bus stops that will be visited, along with a scheduled arrival and departure time, and whether or not each stop is a timepoint (optional). 
A stop_times.txt file will look like the following:

Table 1.1 - An example of data from the GTFS format stop_times.txt file

$\begin{array}{lllll}\text { trip_id } & \text { arrival_time } & \text { departure_time } & \text { stop_id } & \text { stop_sequence } \\ 2777 & 5: 52: 00 & 5: 52: 00 & 4301 & 1 \\ 2777 & 5: 52: 34 & 5: 52: 34 & 3471 & 2 \\ 2777 & 5: 53: 46 & 5: 53: 46 & 4456 & 3 \\ 2777 & 5: 54: 27 & 5: 54: 27 & 592 & 4 \\ 2777 & 5: 55: 11 & 5: 55: 11 & 593 & 5\end{array}$

The GTFS-realtime specification can be broken down into three types of elements:

- $\quad$ Trip Updates - Real-time predictions for when vehicles arrive and depart. Predictions (stop_time_updates) are represented as an update to the time that the vehicle was scheduled to arrive or depart (defined in GTFS stop_times.txt), either as a relative “delay” or "time.” stop_time_updates are identified using a trip ID from GTFS trips.txt.

- Vehicle Positions - Real-time vehicle location, trip assignment (defined using the trip ID from GTFS trips.txt), and occupancy information.

- Service Alerts - Descriptions of events that affect transit service, along with the transit stops/routes that the event impacts. For example, "Route 5 is on detour due to flooding." 
A GTFS-realtime Trip Update for trip_id 2777 that predicts a bus running 60 seconds late for stop_id 4456 (stop_sequence 3), running on time for stop_id 592 (stop_sequence 4), and 60 seconds early for stop_id 593 (stop_sequence 5), would look like the following:

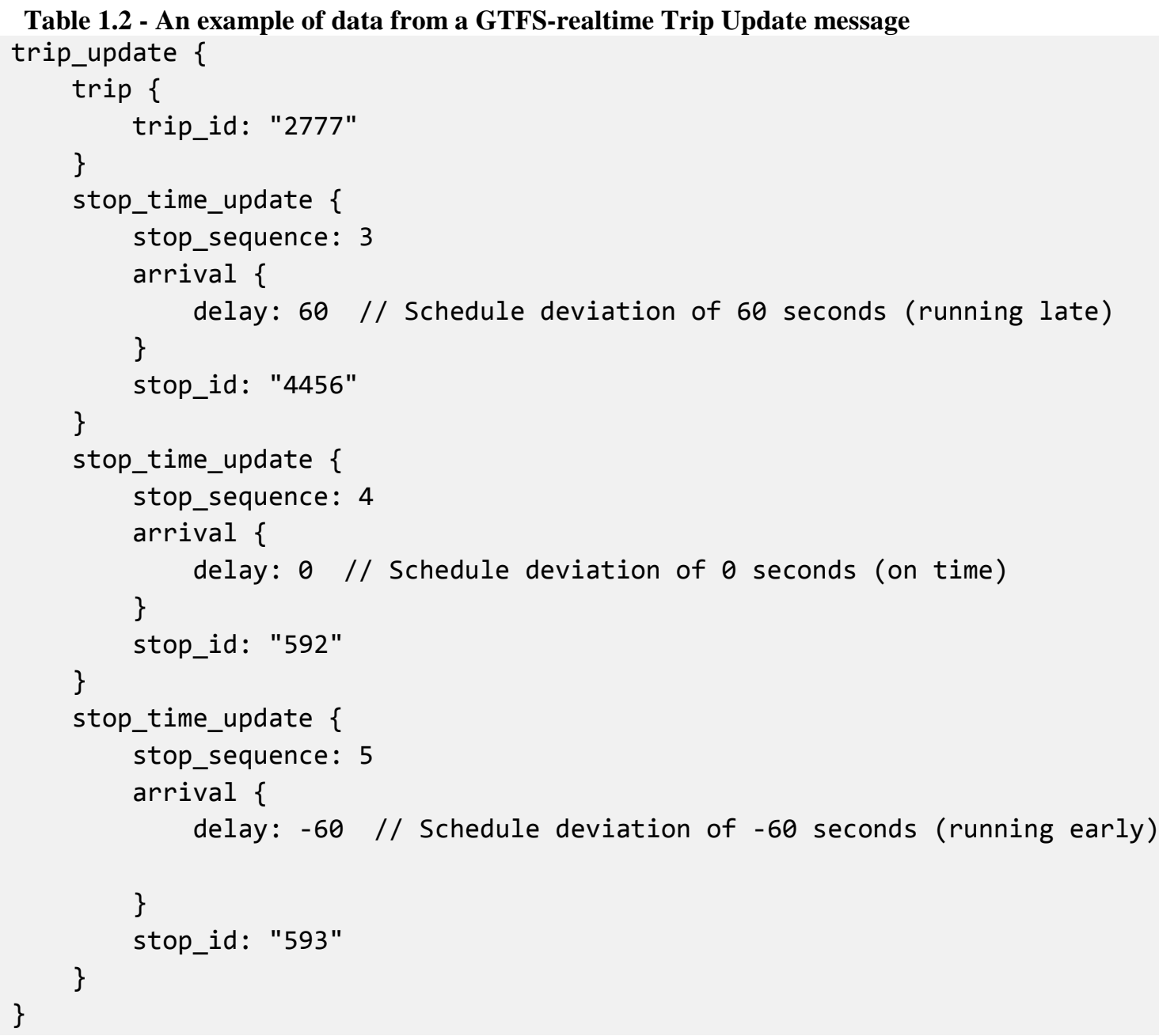

The architecture of a real-time transit information system can be divided up into two components [20] as shown in Figure 1:

1. The Producer - The system generating the GTFS-realtime feed (typically the automatic vehicle location (AVL) system), labeled as the “Transit Agency Server” in Figure 1.

2. The Consumer - The system reading the GTFS-realtime feed (typically a server and mobile app displaying the information to a transit rider), labeled as the "App Developer Server” in Figure 1. 


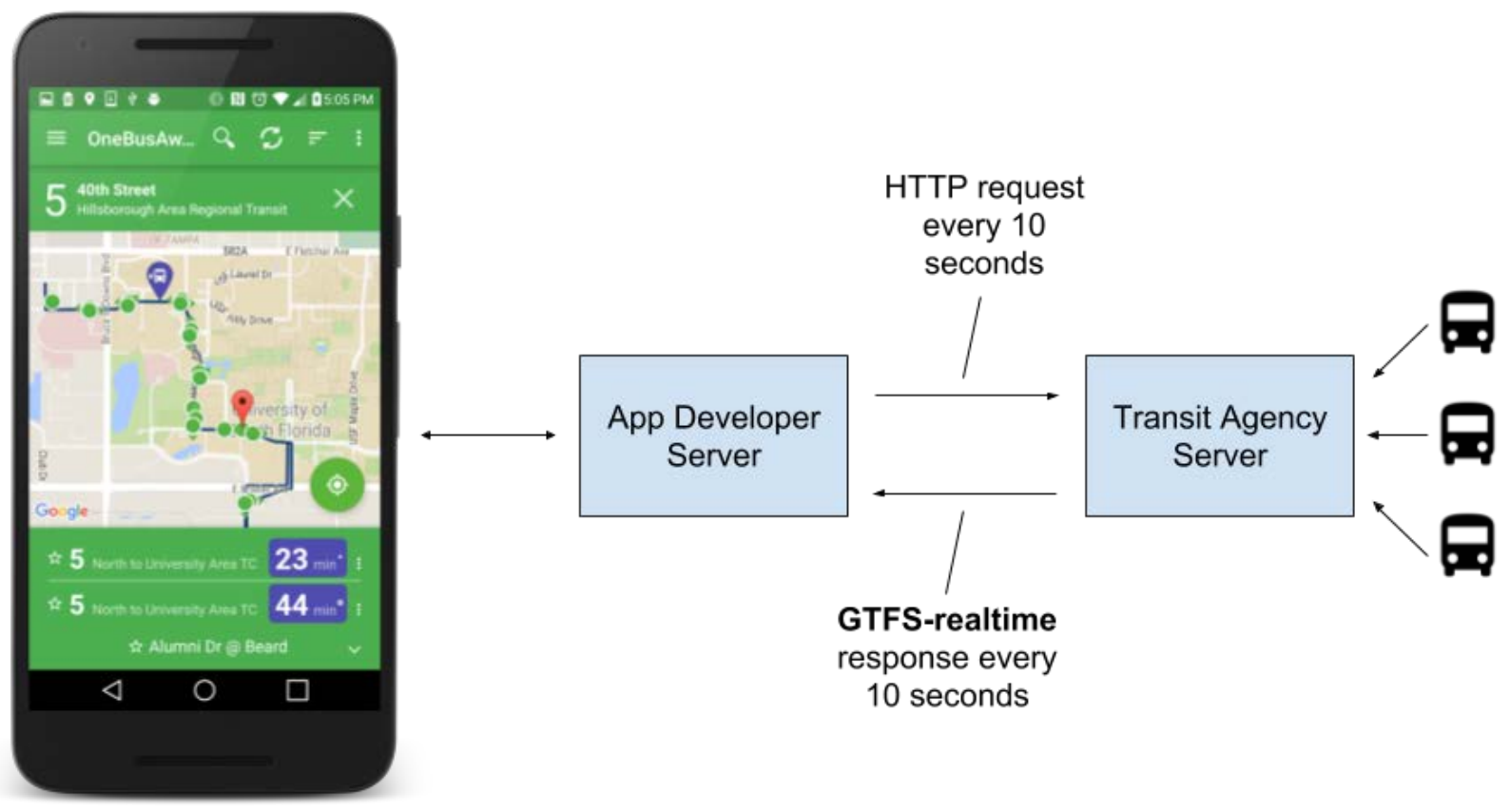

Figure 1 - Typical real-time information flow from a transit agency to a mobile app

While GTFS datasets are typically updated 3-4 times per year (e.g., when new schedules are published), a GTFS-realtime Trip Updates and Vehicle Positions feed can be updated as often as every few seconds and are typically driven by an AVL system.

GTFS-realtime datasets are formatted in the Protocol Buffer format [21], which is a very efficient binary representation of the information in the feed. As a result, the actual GTFSrealtime messages produced and consumed by applications require special software to convert them to human-readable plain text. 


\subsection{GTFS-REALTIME V2.0}

GTFS-realtime v2.0 accomplishes a major step forward in terms of better defining what information should be contained in a GTFS-realtime message. The following section discusses the limitations in GTFS-realtime v1.0 that drove the development of v2.0.

\subsection{CHALLENGES WITH GTFS-REALTIME V1.0}

Having a de facto standard for real-time information is beneficial to those who use the data - it lets app developers focus on creating new features instead of developing convertor software for each feed type and identifying and removing discrepancies in the data.

However, as more transit agencies and app developers started using GTFS-realtime, they noticed something peculiar - almost all of the GTFS-realtime fields were optional. To be exact, of the 63 GTFS-realtime data fields, only seven were required - about $11 \%$ of all fields [10].

The overwhelming number of optional fields makes it very simple for AVL system implementers to roll out a GTFS-realtime feed that is officially compliant with GTFS-realtime v1.0 - they can leave most of the values blank. However, it creates challenges when app developers start consuming that information, as some critical information may be missing. Transit riders are upset when an app gives them bad data. Information errors reflect poorly on the app developer and transit agency, and cause problems with the AVL vendor due to unmet data quality expectations.

As an example, Table 2.1 shows a fully compliant GTFS-realtime v1.0 feed for a vehicle position:

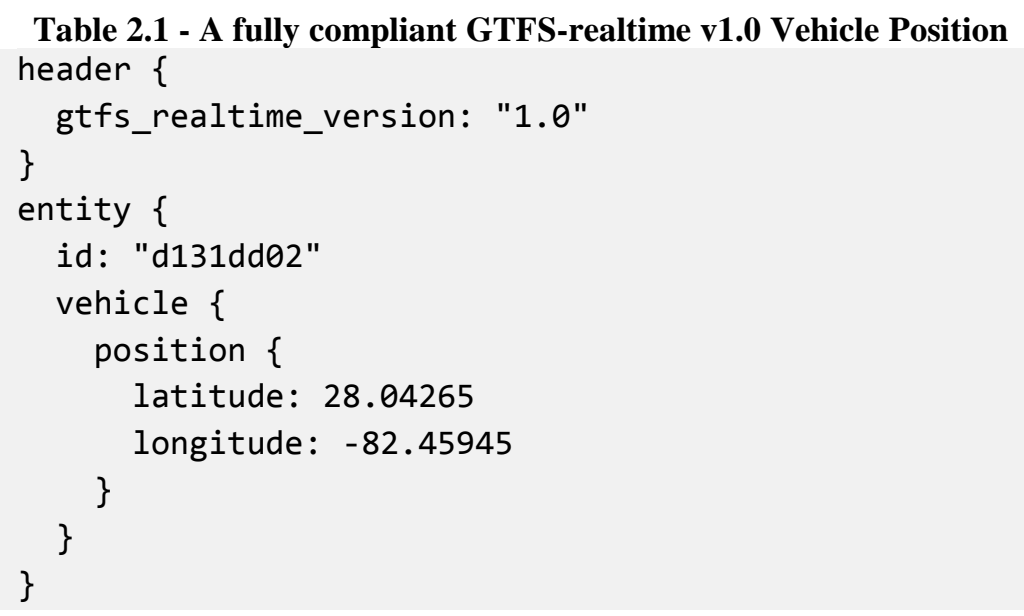


There is critical information missing:

- When was this position calculated?

- What route or trip is this vehicle currently serving?

- How do we describe the vehicle to a transit rider? Is d131dd02 a valid bus number?

A second example is shown in Table 2.2. When providing arrival predictions in GTFS-realtime v1.0, the stop_sequence field is optional:

Table 2.2 - A missing stop_sequence value is problematic for routes with loops

trip \{

trip_id: "277725"

\}

stop_time_update \{

arrival \{

delay: 900 // 15 minutes

\}

stop_id: "A"

\}

This message that contains stop_id, but not stop_sequence, works fine for most routes. However, a missing stop_sequence value creates problems when you have a route with a loop that visits a stop more than once, such as that shown in Figure 2.

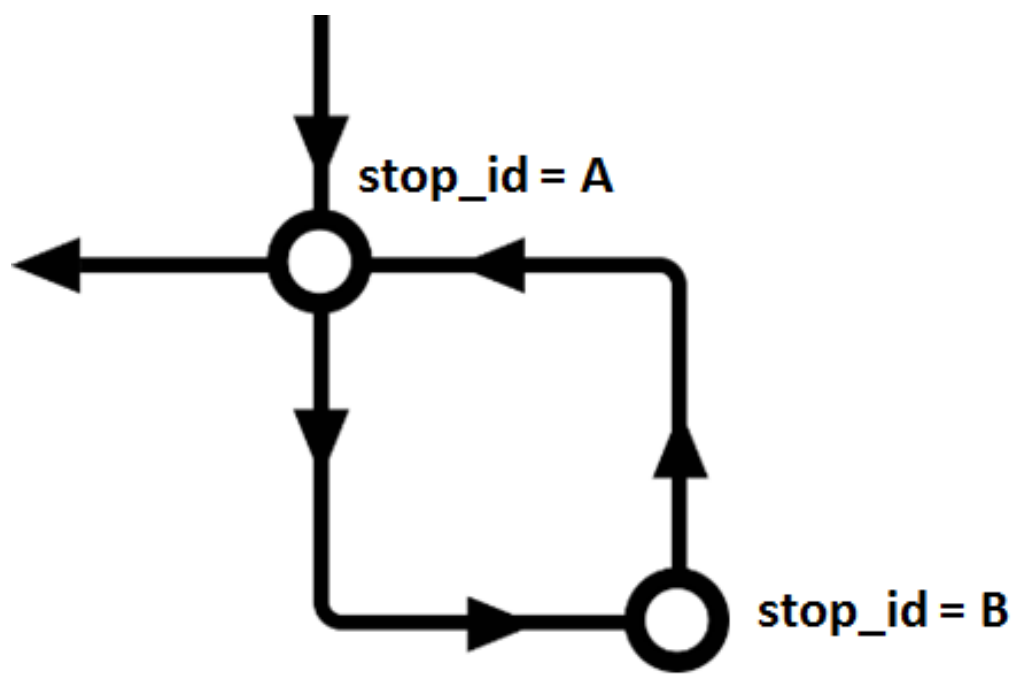

Figure 2 - A route that includes a loop that visits stop_id A more than once 
Without stop_sequence, the delay value of 15 minutes could potentially pertain to either the first time the vehicle arrives at Stop A or the second. This ambiguity creates a problem when the information is shown to riders. Riders waiting to board at Stop B will be upset if the app tells them they have time to grab an extra coffee (because it interprets the 15-minute delay being in the first half of the loop) and then they miss the bus when the vehicle arrives on time because the delay was actually in the second half of the loop. The stop_sequence field is required in this case to interpret the prediction correctly.

The labeling of optional fields in GTFS-realtime v1.0 documentation was driven by the use of Protocol Buffers [21] for compressing real-time data messages. Protocol Buffers are an extremely compact way to represent information in a binary format. Instead of the feed data being formatted as Unicode text characters as shown earlier in this report, each of which takes at least 1 byte ( 8 bits), it can be compressed into a smaller representation of 0 s and 1 s (i.e., bits).

The space savings of Protocol Buffers adds up quickly, especially considering that GTFSrealtime messages are exchanged every few seconds. For example, a single response from Massachusetts Bay Transportation Authority (MBTA)'s GTFS-realtime Trip Updates feed in the binary Protocol Buffer format is under $1 \mathrm{MB}$, while the plain text version is more than six times bigger at just over 5.5 MB.

\begin{tabular}{|llr|}
\hline Name & Type & \multicolumn{1}{r|}{ Size } \\
[ TripUpdates.pb & PB File & $891 \mathrm{~KB}$ \\
TripUpdates.pb.txt & Text Document & $5,683 \mathrm{~KB}$ \\
\hline
\end{tabular}

Figure 3 - An example of the space savings of the binary Protocol Buffer format

While binary formats are extremely space efficient, it can be time consuming to create software that processes them. Protocol Buffers solve this problem by generating this code automatically. First, the developer creates a proto file that describes the data elements to exchange (in the case of GTFS-realtime, the official gtfs-realtime.proto, shown in Table 2.3). 


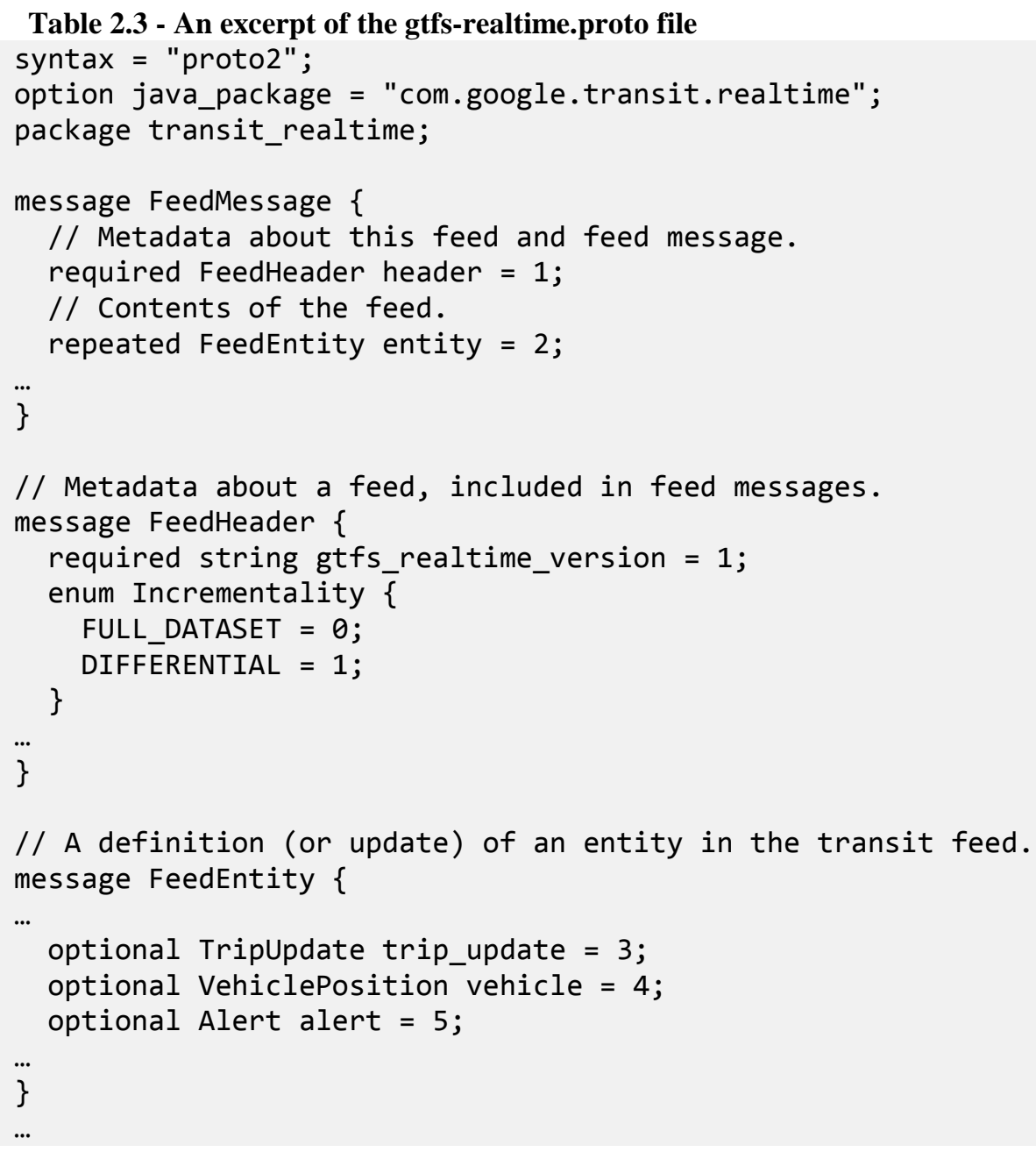

Then, developers can input this .proto file into the open-source Protocol Buffer tools [21] to autogenerate the code that compresses the information to a binary format (used in the Transit Agency AVL server) and the code to extract it from a binary format (used in the App Developer's server), as shown in Figure 4. 


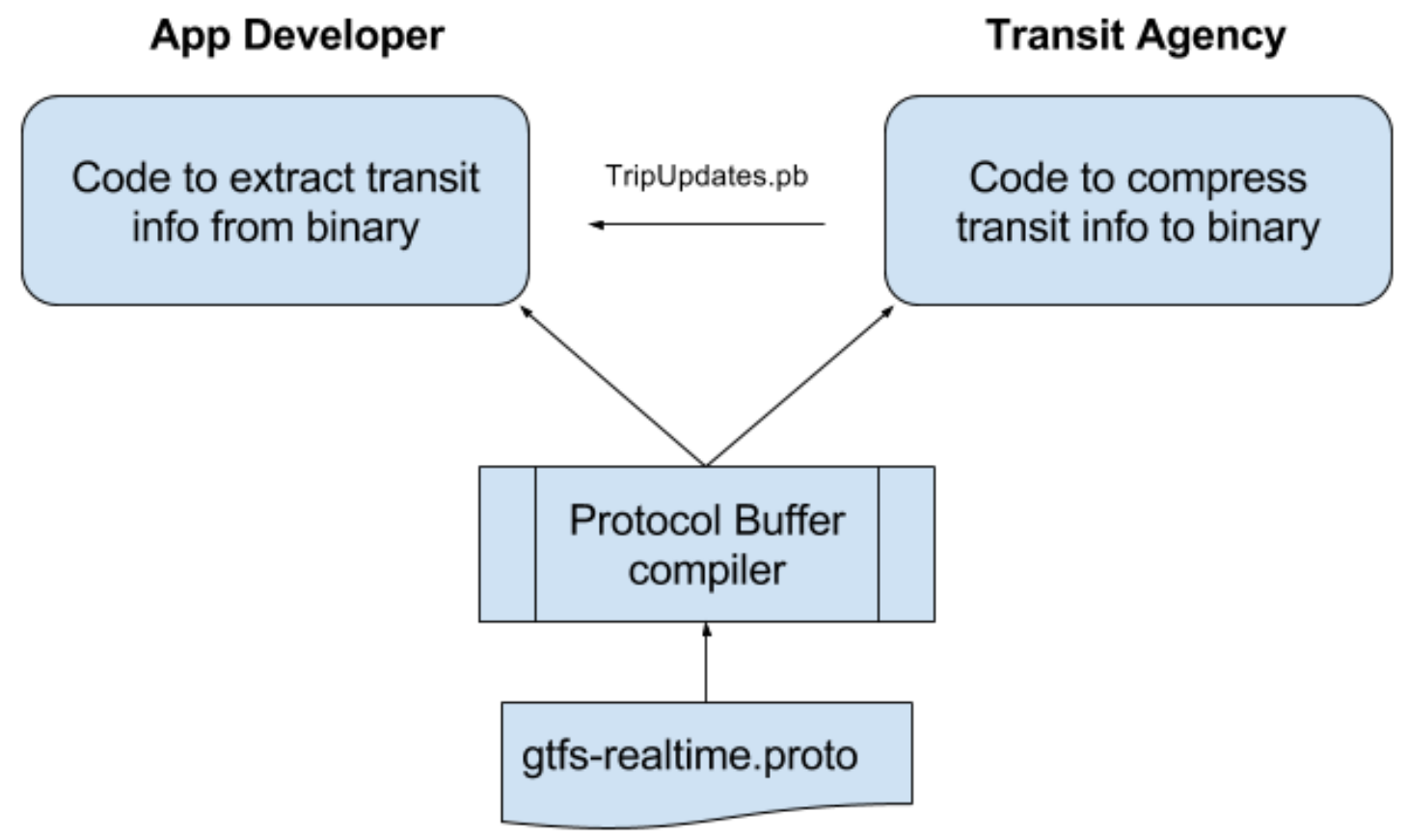

Figure 4 - The Protocol Buffer compiler autogenerates code to exchange binary GTFS-realtime messages

To make this process even more convenient, Google has already performed these steps and created a readily usable gtfs-realtime-bindings library [22] that supports easily exchanging GTFS-realtime messages in the programming languages Java, .NET, JavaScript / Node.js, PHP, Python, Ruby, and Golang.

The implementation of GTFS-realtime in the Protocol Buffer format was the reason behind the extensive number of fields marked "Optional" in GTFS-realtime v1.0. The GTFS-realtime spec includes a Cardinality field that was copied from the gtfs-realtime.proto file and does not have anything to do with public transportation. Protocol Buffer Cardinality simply defines whether or not software parsing the binary message expects a field to exist - it has no direct mapping to GTFS or transit-specific logic (repeated, in case you're wondering, is Protocol Buffer-lingo for a list of optional elements). This becomes a problem in GTFS-realtime because many software engineers choose not to label any Protocol Buffer fields as Required because of forward compatibility issues with Protocol Buffer implementations (see the Protocol Buffer documentation "Required is Forever" [23] and a GTFS-realtime Google Group discussion [24] for details). As a result, nearly all fields in GTFS-realtime v1.0 are shown as "Optional," even if that field is necessary for a transit app to show proper real-time information to a transit rider. 


\subsection{DEFINING TRANSIT-SPECIFIC FIELD REQUIREMENTS}

The research team worked with the GTFS-realtime community to create an updated version of the format that defines the semantic requirements and cardinality of real-time information to fix this confusion about which fields are optional and required. GTFS-realtime v2.0 [10] now defines which fields should be required based on domain-specific (i.e., transit) logic. The detailed proposal for defining semantic cardinality is available online [25]. These definitions are completely independent of the Protocol Buffer format and would still apply even if a different format was used for GTFS-realtime data.

In GTFS-realtime v2.0, each field now has a Required column that can contain the following values:

- $\quad$ Required: This field must be provided by a GTFS-realtime feed producer.

- Conditionally required: This field is required under certain conditions, which are outlined in the field Description. Outside of these conditions, the field is optional.

- Optional: This field is optional and is not required to be implemented by producers. However, if the data is available in the underlying automatic vehicle location systems (e.g., VehiclePosition timestamp) it is recommended that producers provide these optional fields when possible.

The Cardinality column now represents the number of elements that can be provided for a particular field - One or Many (e.g., a list of predictions applying to more than one stop within a trip).

Below in Figure 5 is a snapshot of what the GTFS-realtime FeedHeader looks like in GTFSrealtime v1.0 (top), and GTFS-realtime v2.0 (bottom): 


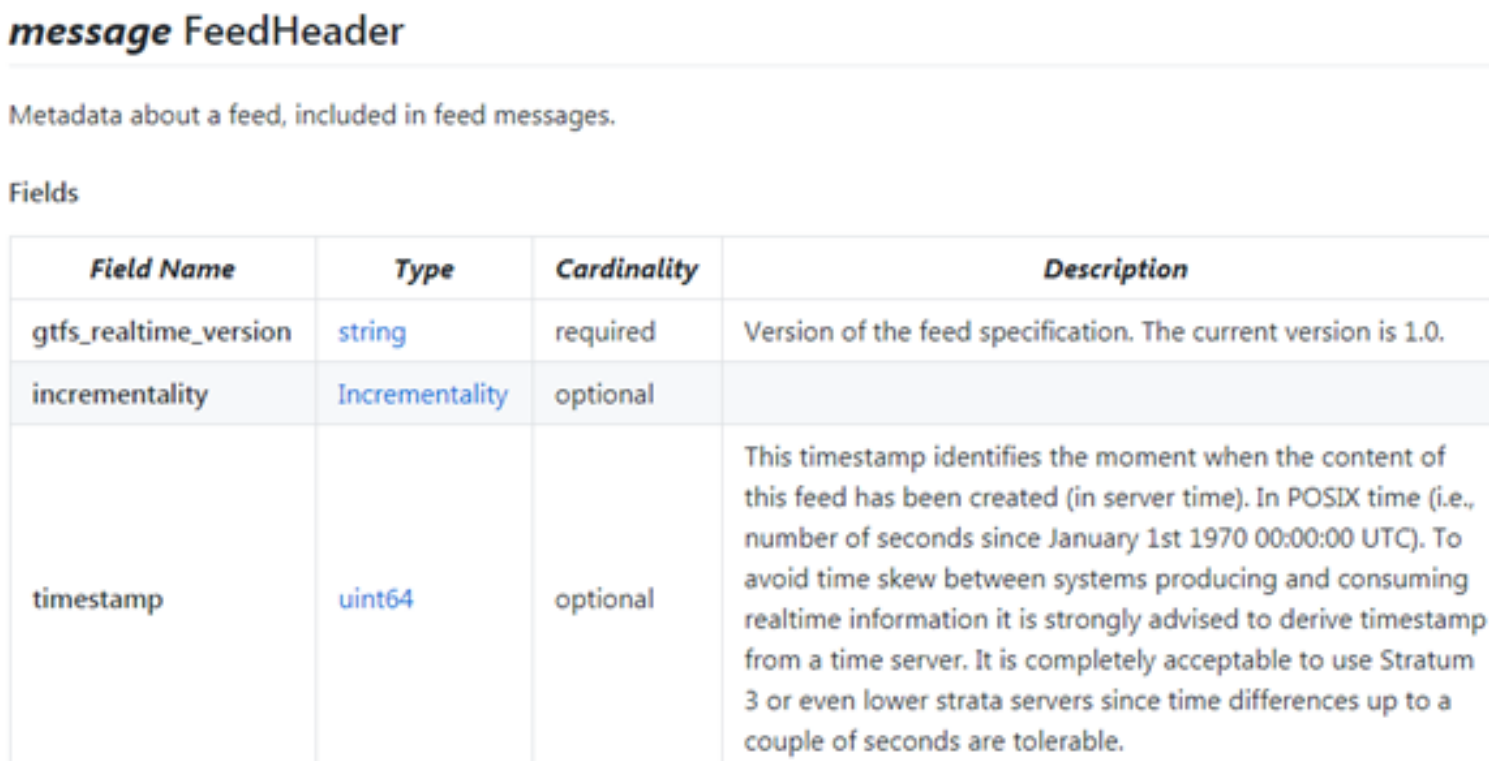

This timestamp identifies the moment when the content of this feed has been created (in server time). In POSDX time (i.e., number of seconds since January 1st 1970 00:00:00 UTC). To avoid time skew between systems producing and consuming realtime information it is strongly advised to derive timestamp from a time server. It is completely acceptable to use Stratum 3 or even lower strata servers since time differences up to a couple of seconds are tolerable.

\section{GTFS-realtime v1.0}

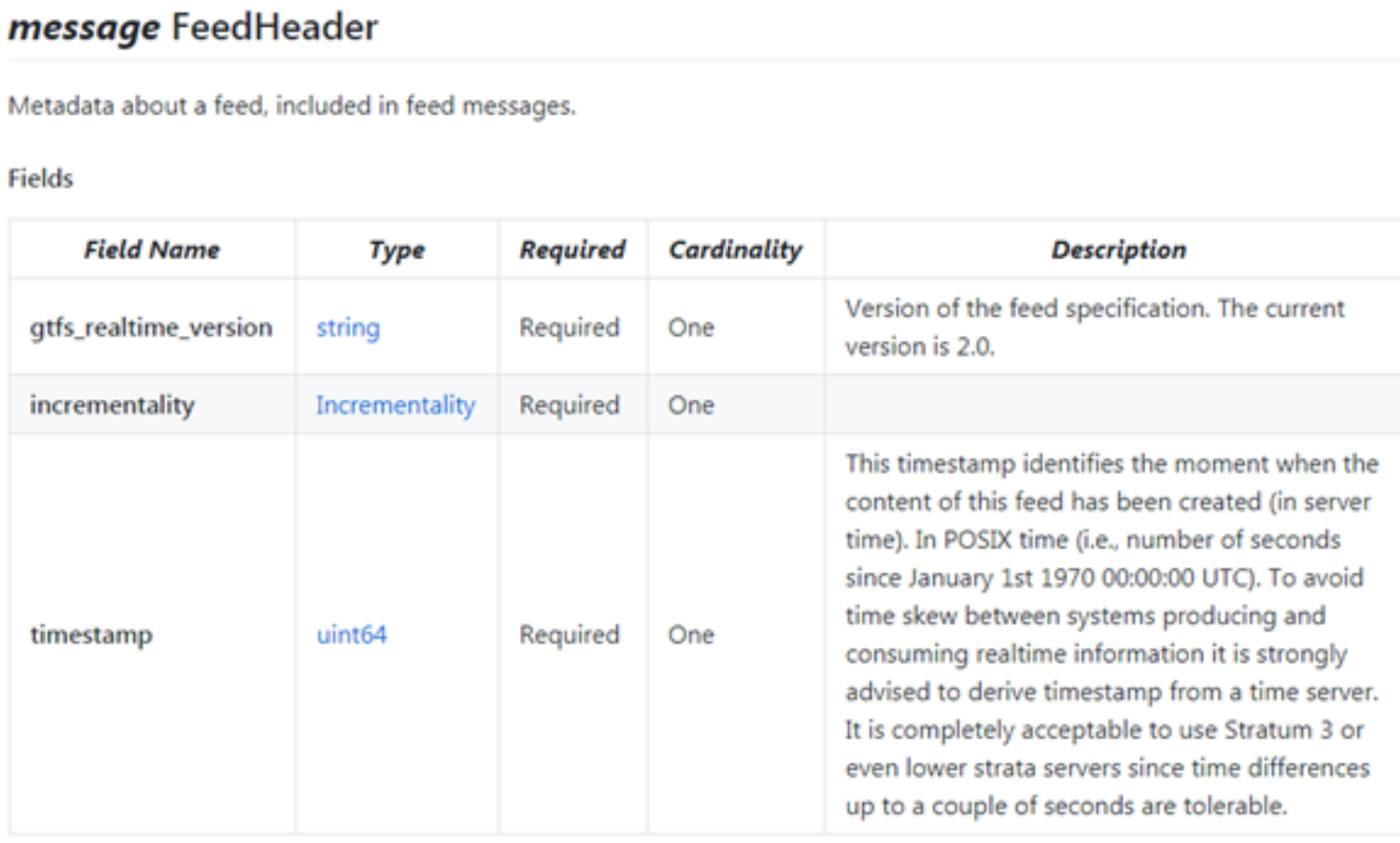

\begin{tabular}{|c|c|c|c|c|}
\hline Field Name & Type & Required & Cardinality & Description \\
\hline gtfs_realtime_version & string & Required & One & $\begin{array}{l}\text { Version of the feed specification. The current } \\
\text { version is } 2.0 \text {. }\end{array}$ \\
\hline incrementality & Incrementality & Required & One & \\
\hline timestamp & uint64 & Required & One & $\begin{array}{l}\text { This timestamp identifies the moment when the } \\
\text { content of this feed has been created (in server } \\
\text { time). In POSIX time (i.e., number of seconds } \\
\text { since January } 1 \text { st } 197000: 00: 00 \text { UTC). To avoid } \\
\text { time skew between systems producing and } \\
\text { consuming realtime information it is strongly } \\
\text { advised to derive timestamp from a time server. } \\
\text { It is completely acceptable to use Stratum } 3 \text { or } \\
\text { even lower strata servers since time differences } \\
\text { up to a couple of seconds are tolerable. }\end{array}$ \\
\hline
\end{tabular}

\section{GTFS-realtime v2.0}

Figure 5 - Example of GTFS-realtime v2.0 showing new "Required” and "Cardinality” fields 
The new Required column can be seen in the v2.0 version of the documentation, which now makes critical fields like the header timestamp mandatory. This helps address the previously discussed problem of determining the age of a vehicle position.

The StopTimeUpdate message, which contains the information about arrival and departure predictions, is a good illustration of the new Conditionally required value:

\section{message StopTimeUpdate}

Realtime update for arrival and/or departure events for a given stop on a trip. Please also refer to the general discussion of stop time updates in the TripDescriptor and trip updates entities documentation.

Updates can be supplied for both past and future events. The producer is allowed, although not required, to drop past events. The update is linked to a specific stop either through stop_sequence or stop_id, so one of these fields must necessarily be set. If the same stop_id is visited more than once in a trip, then stop_sequence should be provided in all StopTimeUpdates for that stop_id on that trip.

Fields

\begin{tabular}{|l|l|l|l|}
\hline Field Name & Required & Cardinality & Description \\
\hline & & Must be the same as in stop_times.txt \\
in the corresponding GTFS feed. \\
stop_sequence
\end{tabular}

Figure 6 - The stop_sequence field as defined in GTFS-realtime v2.0

In GTFS-realtime v2.0, stop_sequence is Conditionally required, and one of the required cases outlined in the Description is the loop, which addresses the problem with the ambiguous arrival prediction for the loop route presented earlier. 


\subsection{IMPROVING GTFS DATA UNIFORMITY}

In parallel to the development of the GTFS-realtime v2.0, the research team contributed to an effort organized by the Rocky Mountain Institute to develop a set of GTFS Best Practices [26]. This effort attempted to address shared challenges that transit app developers encounter when working with GTFS data. Because GTFS-realtime requires GTFS data, these issues also impact the quality of a GTFS-realtime feed as well.

\subsection{CHALLENGES OF GTFS DATA UNIFORMITY}

Following are some examples of challenges that transit app developers have encountered in the past when trying to process GTFS data for a large number of sources.

\subsubsection{Feed management}

- Changing GTFS dataset URLs - In order to manage a large number of agencies in an app, app developers typically create software that automates the process of retrieving new GTFS datasets from agencies and integrating them into their application. If an agency puts their GTFS zip file at the location http://acmeagency.org/gtfs.zip, app developers will assume that new GTFS datasets will be uploaded to the same URL and will continuously poll that URL to see if the data changed. If an agency changes this URL for a new dataset (http://acmeagency.org/gtfs-March-2017.zip), the app will never know that this new data exists.

- Changing GTFS dataset IDs - A typical feature in transit apps are stop bookmarks so a user can easily retrieve information about their favorite stops. The app developer needs to have a way to track which stops the user has marked as their favorites. A simple way to do this is to save the stop_id of that stop to a database. Then, the next time the user wants to see arrival information for that stop, the saved stop_id is used to fetch real-time arrival information. This works well, until the next GTFS update when the stop_id for that stop changes. Now the user can't retrieve arrival information for their favorite stop because that stop_id no longer exists.

- Gaps in GTFS data coverage-Agencies typically update their GTFS data on a quarterly basis. Therefore, agencies wait to do this until just before their previous data expires. For example, if changes go into effect April 1, an agency may publish their new GTFS dataset in the late hours of March 31. To make this data available to their users, app developers need to download the new dataset, validate it, add the new data to their 
databases, and potentially push an update to all applications. This can definitely take more than a few hours to accomplish, which means that on April 1, when the user opens the app, no transit service will be visible. Agencies are able to avoid this issue by using the GTFS Merge Tool [27] to create a combined dataset for both the current and next schedule period, and by sharing/announcing this merged dataset to developers at least a week prior to the service change. This way, apps pick up the schedule change early and are ready to go when the change actually happens.

\subsubsection{Data content}

- Case of text-Some agencies PUBLISH ALL THEIR STOP NAMES, ROUTE NAMES, AND HEADSIGNS IN ALL CAPS! This makes text difficult to visually read in the app, and also makes it hard to distinguish between abbreviations and words.

- Abbreviations-When text is abbreviated, it's meaning is not always clear. For example, is "Dr." for "Doctor" or "Drive"? Sometimes we can tell the difference visually in context, but this is particularly challenging for text-to-speech engines (e.g., for accessibility, voice-driven personal assistants such as Amazon Alexa, Google Assistant, and Siri).

- Loop routes - If there is a route that continuously runs in a circle, should the first/last stop be included in the trip twice, or just once? The GTFS community found that the majority represent it with the same stop twice, once at the beginning of the trip and again at the end, but not everyone represents their data this way.

\subsection{GTFS BEST PRACTICES}

GTFS differs from other data standards in that it is largely a grassroots effort driven by transit agencies that produce the data as well as application developers who consume it in their applications - there is no official balloted standards organization (e.g., APTA, IEEE, ISO) that controls the format. GTFS has a community-driven process to adopt changes to the format, where anyone can propose a change (e.g., the changes for GTFS-realtime v2.0) that is then voted on by others in the community. However, there is a high bar for adoption of changes - a change must be implemented by both an agency as well as an application developer before a vote can be opened. Additionally, for a change to be adopted the vote must have unanimous approval - any votes against the proposal will prevent it from being adopted.

While the above governance model has served GTFS well in keeping it focused on a core set of services surrounding customer-facing trip planning and real-time information systems, it has resulted in some useful ideas that are endorsed by the majority, but not all, of those using GTFS being left out of the GTFS documentation. The research team collaborated with some of the 
most active members in the GTFS community [11] to create the GTFS Best Practices (http://gtfs.org/best-practices/) that are based on these concepts.

GTFS Best Practices help address some of the major challenges in data fragmentation (including the above-mentioned items) and provide guidance to transit agencies and vendors/consultants helping to produce and consume GTFS data, as well as app developers that make the information available in their applications. GTFS Best Practices differ from the GTFS reference specification in that Best Practices only require a majority vote for adoption, while changes to the GTFS reference specification require unanimous consent. Additionally, GTFS Best Practices include guidance on how a feed should be managed and updated by a transit agency, which is beyond the scope of the GTFS reference specification that only governs the data format.

The GTFS Best Practices documentation is organized both by file from the GTFS dataset as well as by various use cases such as trip planners, human readability, arrival predictions, and timetables. This makes it simple for agencies and developers to understand what best practices should be followed for each file in the dataset, as well as the implications that various practices have on certain types of applications.

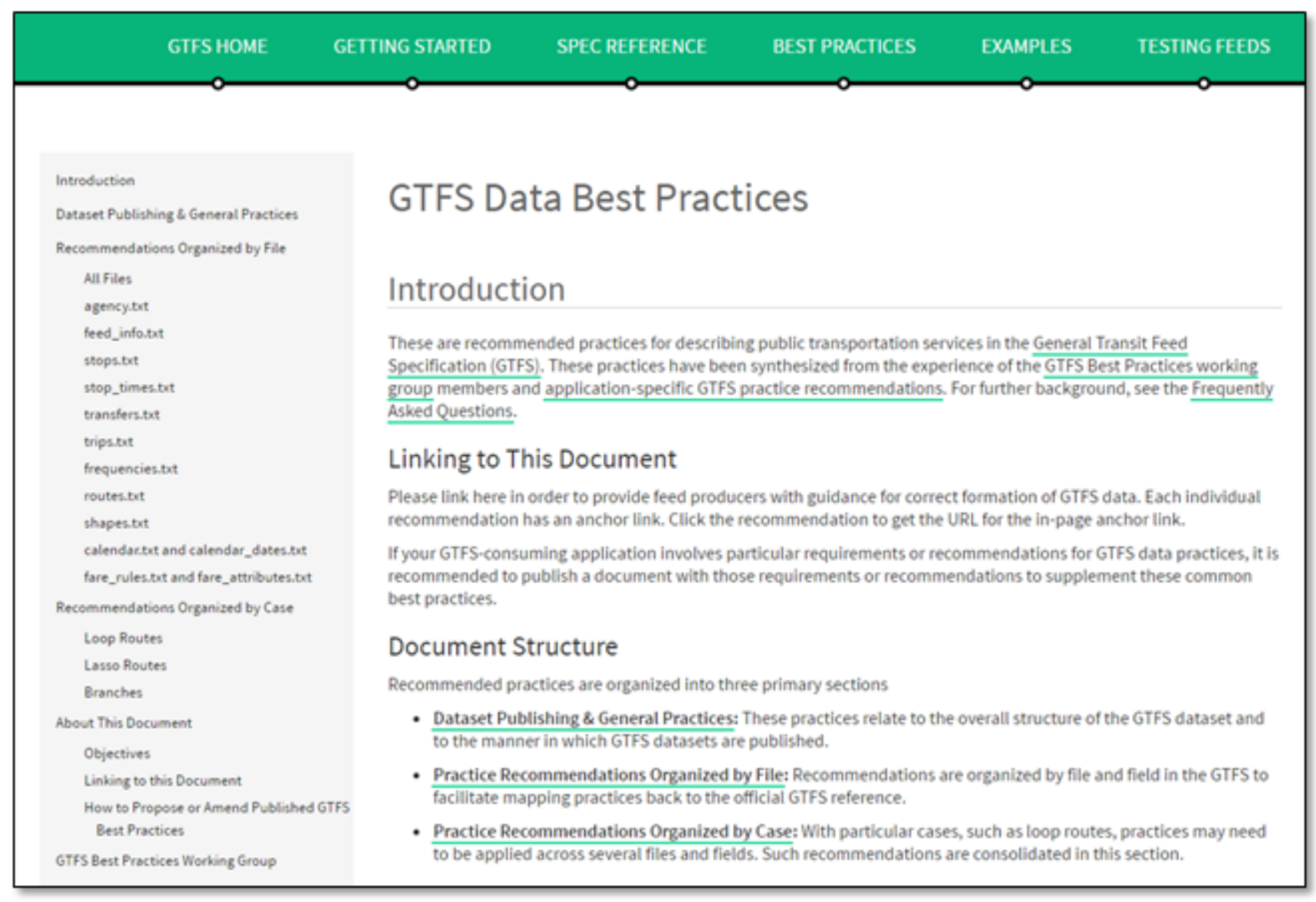

Figure 7 - The new GTFS Best Practices, available at http://gtfs.org/best-practices/ 


\subsection{ADDITIONAL GTFS AND GTFS-REALTIME IMPROVEMENTS}

In addition to the GTFS Best Practices, the research team also proposed several other improvements to the GTFS and GTFS-realtime formats and governance process that were voted on and accepted by the GTFS community, including the following:

- Clarify that a proposer's vote does not count towards total votes https://github.com/google/transit/pull/50

- Specify that prior to a vote a GTFS producer/consumer should implement change https://github.com/google/transit/pull/46

- Better cross-linking for a pull request and Google Groups post https://github.com/google/transit/pull/35

- Add missing agency_email field - https://github.com/google/transit/pull/34 


\subsection{GTFS-REALTIME VALIDATION TOOL}

While the GTFS format for schedule data has several open-source GTFS feed validators [28], no such open validation tool has existed for GTFS-realtime. The scale of the combined GTFS and GTFS-realtime datasets combined with the frequent refresh of real-time data makes manual inspection time and cost prohibitive. For example, in November 2017 Massachusetts Bay Transportation Authority (MBTA) in Boston [29] had a GTFS dataset that contained 71,260 trips and 1,809,833 stop time records. MBTA's GTFS-realtime feed contains data for 489 vehicles with independent arrival or departure predictions for most stops on active trips that is refreshed around every five seconds. To effectively analyze this quantity and frequency of data, automated tools are required.

\subsection{GTFS-REALTIME VALIDATOR}

To address these problems, the research team created an open-source GTFS-realtime Validator software tool [30] that can monitor GTFS-realtime feeds (Trip Updates, Vehicle Positions, Service Alerts) and log any encountered problems. Instructions for running the GTFS-realtime Validator on any computer are found on the project README page on GitHub [30] (Figure 8).

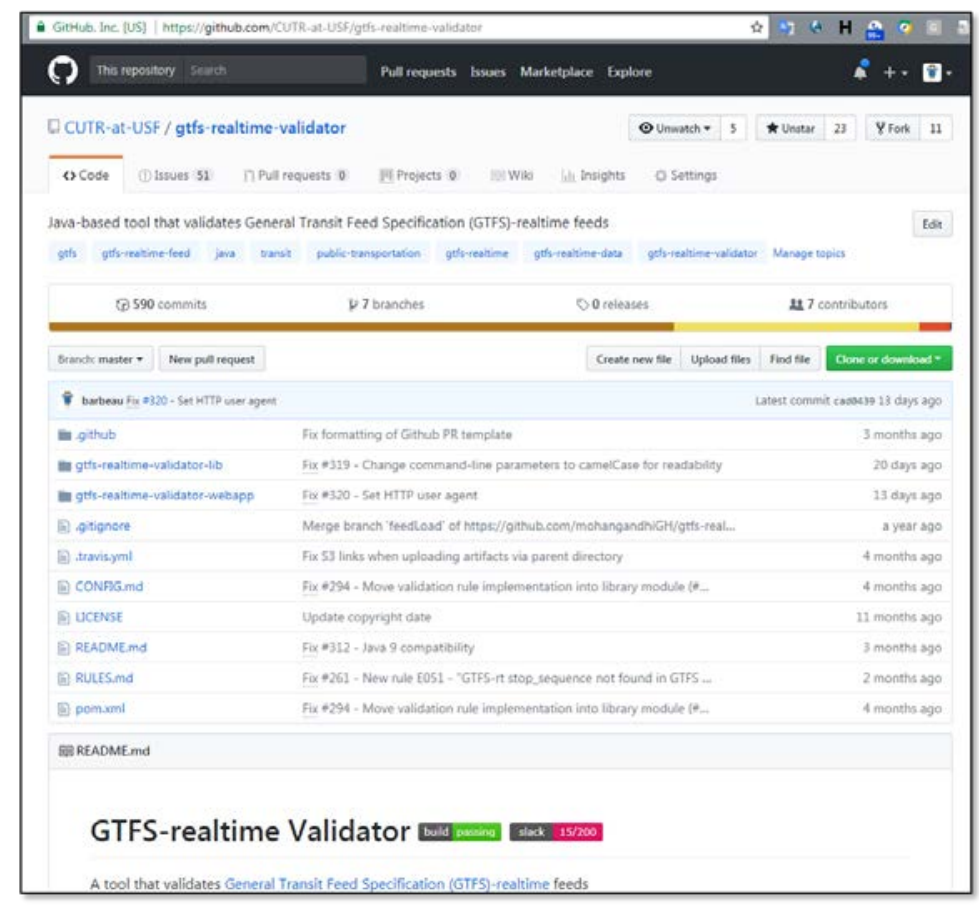

Figure 8 - GTFS-realtime Validator tool source code on GitHub 
To use the GTFS-realtime Validator, as shown in Figure 9, the user simply enters URLs for their GTFS and GTFS-realtime datasets, as well as how frequently the tool should fetch GTFSrealtime updates (the default is 10 seconds) and then click the "Start" button.

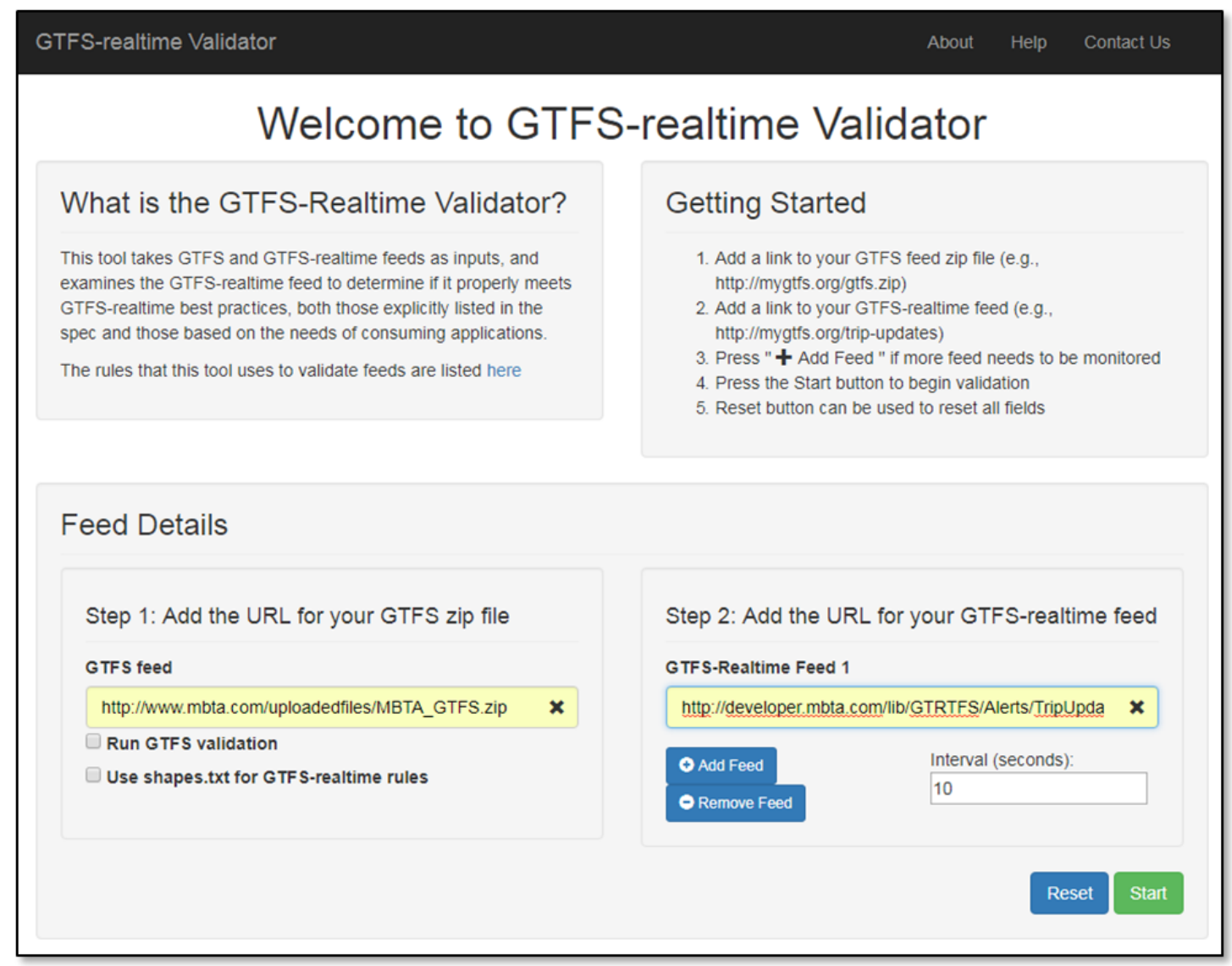

Figure 9 - The first screen of the GTFS-realtime Validator

After starting the monitoring session, the user is shown a "Monitoring Feeds" screen with three main sections: Overview, Summary, and Log (Figure 10). 


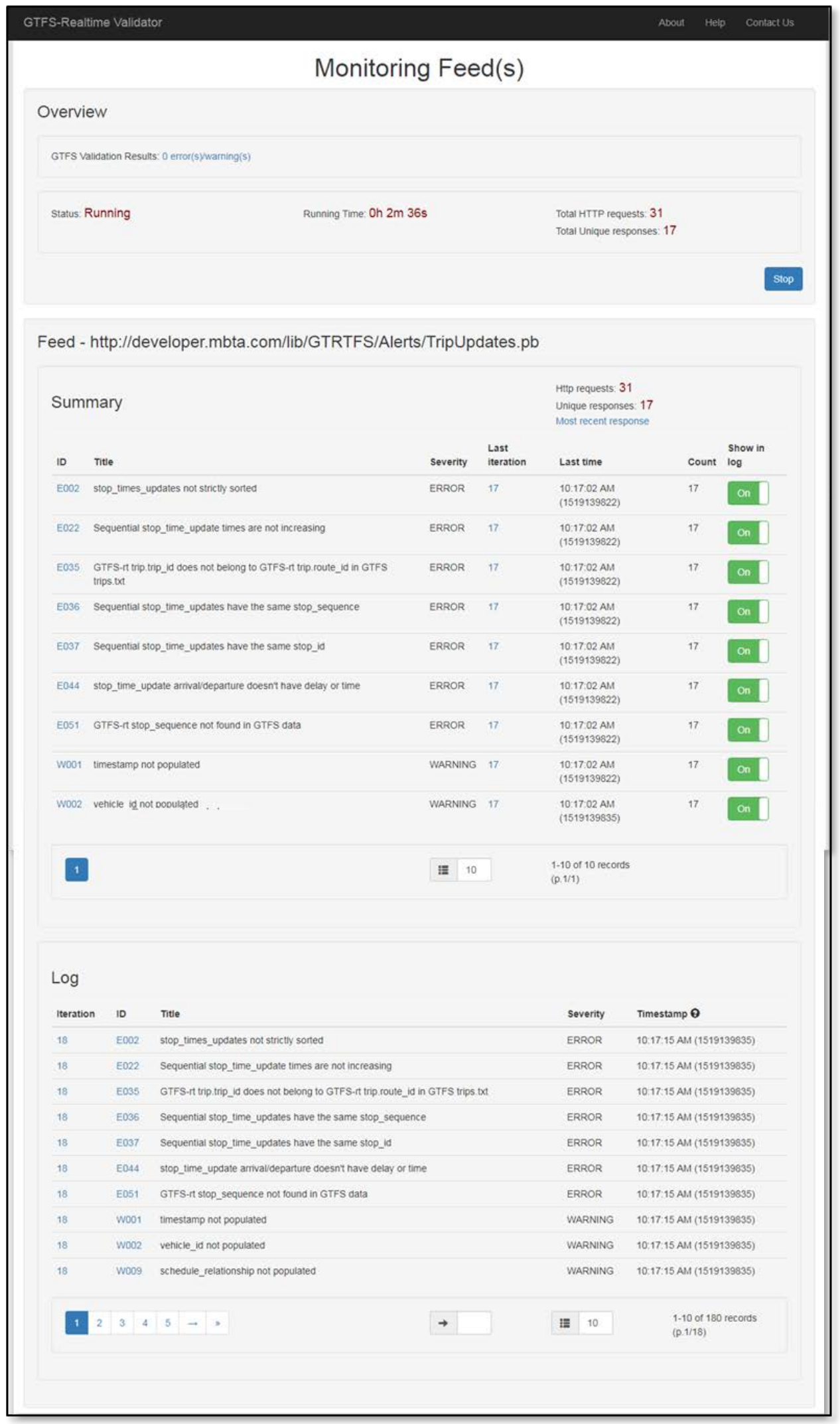

Figure 10 - The monitoring screen, with a summary of all errors and a log of each feed iteration 
The Overview screen provides basic information about how long the monitoring session has been running, along with the number of requests the Validator has made for GTFS-realtime feed updates as well as the number of unique responses that have been returned. If the Validator is polling the GTFS-realtime feed more quickly than it is updated, then duplicate responses will be returned to the Validator. In this case, the validator will detect the duplicate response and will not validate the same response more than once.

Upon each unique response from the GTFS-realtime server, each error and warning that is detected will be output to the Log section. The Log section serves as a historical record of all responses from the server in this session in reverse chronological order. The Summary section is shown above the Log. The Summary has one record for each error or warning that has been detected throughout this monitoring session, along with a count of the occurrences of this error or warning and a link to the last iteration that the error or warning was detected within. On the right side of the Summary section, each error or warning has an on/off switch for "Show in log." The user can toggle this switch to filter our errors or warnings from the Log section that they do not want to see.

In both the Log and Summary sections, all error and warning IDs (e.g., E002) are hyperlinked (Figure 11).

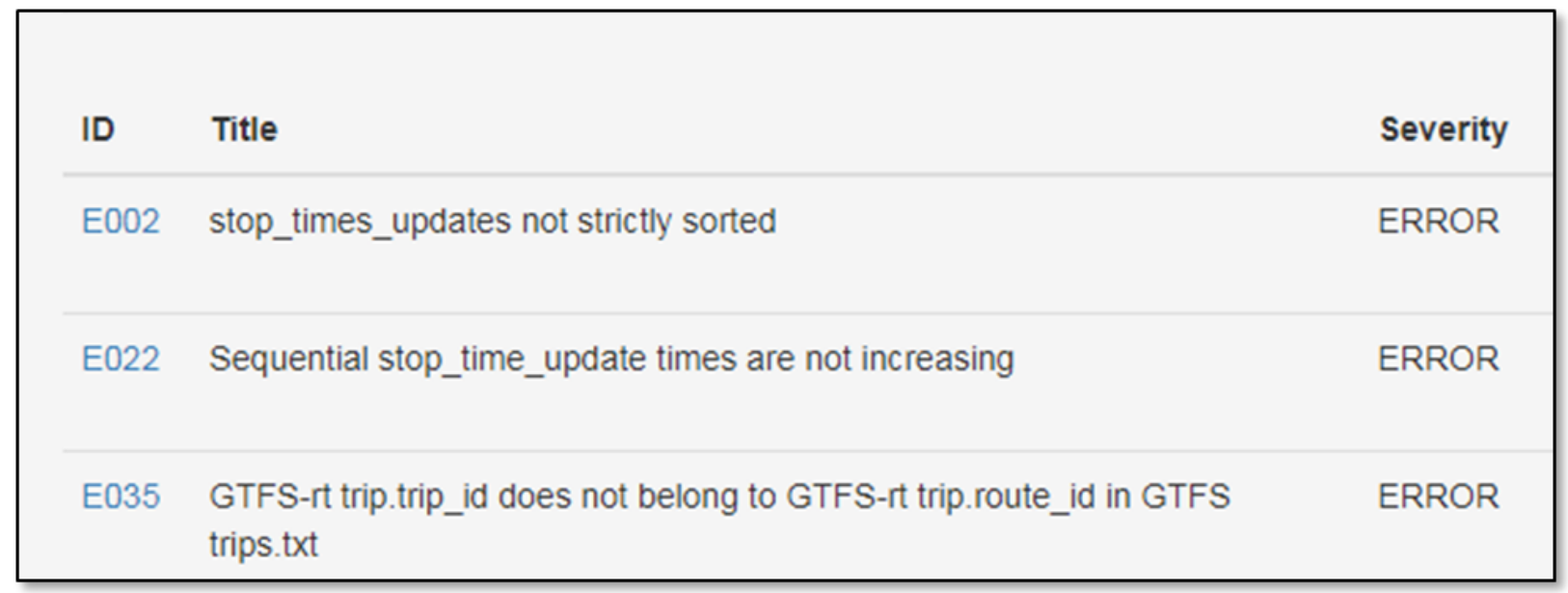

Figure 11 - Error and warning IDs (e.g., E002) shown next to every record in the Summary and Log sections

When clicking on the error or warning ID, the user is directed to the "Implemented Rules" documentation on GitHub [31] where they can read more about that specific error or warning as well as follow reference links to sections within the GTFS-realtime specification related to each rule (Figure 12). For some rules, common mistakes that can cause a particular error are identified and possible solutions that can eliminate the error are provided. 
E001 - Not in POSIX time

All times and timestamps must be in POSIX time (i.e., number of seconds since January 1st 1970 00:00:00 UTC).

Common mistakes - Accidentally using Java's System.currentTimeMillis(), which is the number of milliseconds since January 1st 1970 00:00:00 UTC.

Possible solution - Use TimeUnit.MILLISECONDS. toSeconds(System. currentTimeMillis()) to convert from milliseconds to seconds.

References:

- header.timestamp

- trip_update.timestamp

- vehicle_postion.timestamp

- stop_time_update.arrival/departure.time

- alert.active_period.start and alert.active_period.end

E002 - stop_time_updates not strictly sorted

stop_time_updates for a given trip_id must be strictly ordered by increasing stop_sequence - this also means that no stop_sequence should be repeated.

From Stop Time Updates description:

Updates should be sorted by stop_sequence (or stop_ids in the order they occur in the trip).

From GTFS stop_times.txt :

The values for stop_sequence must be non-negative integers, and they must increase along the trip.

This validation rule is implemented for both when stop_sequence is provided in the GTFS-rt feed, and when stop_sequence is omitted from the GTFS-rt feed.

Common mistakes - Assuming that the GTFS stop_times.txt file will be grouped by trip_id and sorted by stop_sequence while sorting the data is a good practice, it's not strictly required by the spec.

Possible solution - Group the GTFS stop_times.txt records by trip_id and sort by stop_sequence. Also, make sure that no stop_sequence is repeated in GTFS stop_times.txt.

Figure 12 - Two rules from the validator rule documentation on GitHub

\begin{tabular}{|c|c|c|}
\hline Severity & $\begin{array}{l}\text { Last } \\
\text { iteration }\end{array}$ & Last time \\
\hline ERROR & 17 & $\begin{array}{l}\text { 10:17:02 AM } \\
(1519139822)\end{array}$ \\
\hline ERROR & 17 & $\begin{array}{l}\text { 10:17:02 AM } \\
(1519139822)\end{array}$ \\
\hline
\end{tabular}

Figure 13 - The validator screen showing the ID of the last iteration and the time when it was validated 
In the Log and Summary sections, the Iteration ID for the GTFS-realtime message where the error or warning was detected is also hyperlinked (Figure 13). The user can click on this Iteration ID to see detailed information about this GTFS-realtime message, as shown in Figure 14.

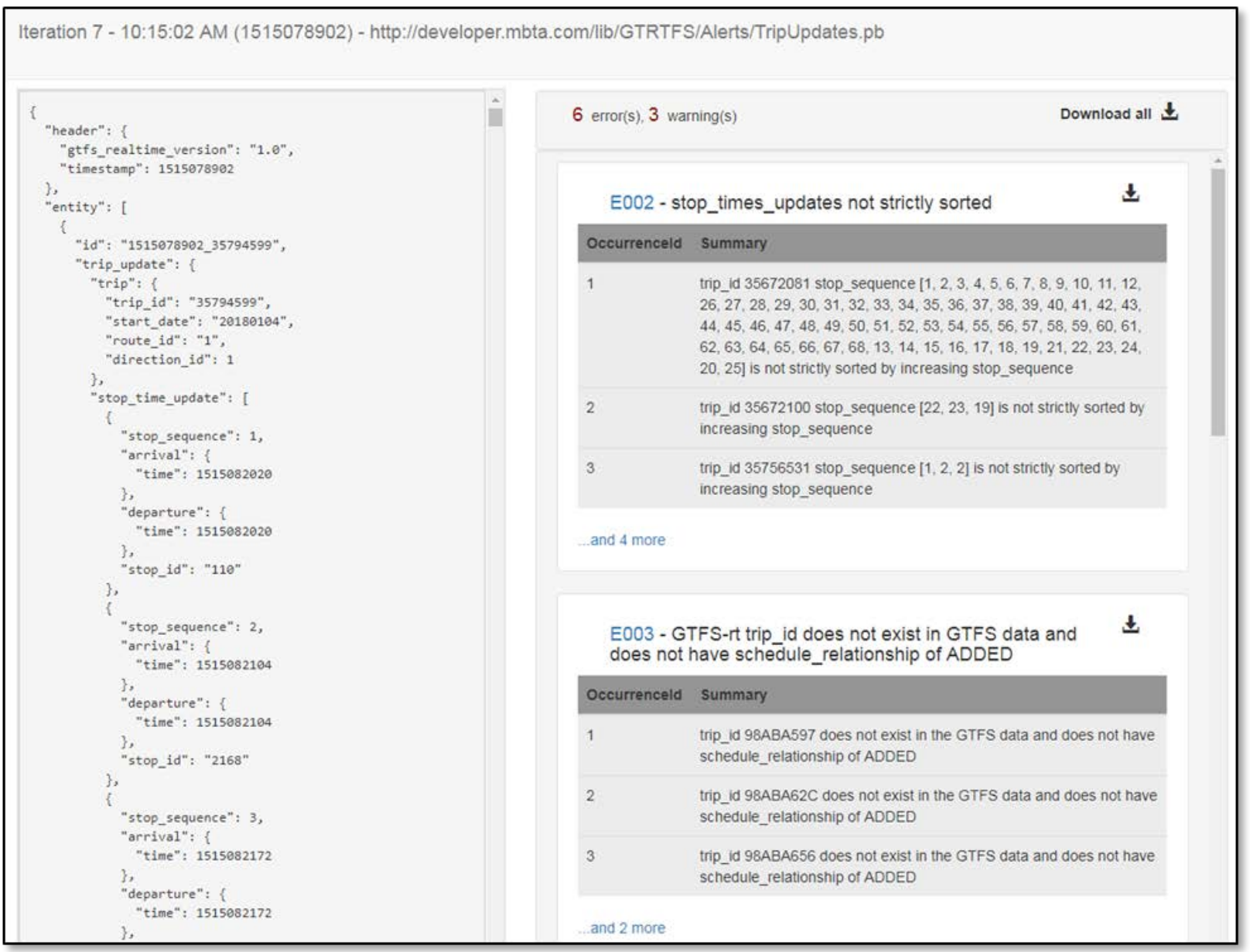

Figure 14 - The Iteration Details screen shows the GTFS-realtime message (left) and occurrences of each error or warning (right)

The GTFS-realtime message that was retrieved from the feed for this iteration is shown on the left, and the occurrences of each error and warning are shown on the right. For example, the user can see that there is a total of seven occurrences of E002 "stop_time_updates not strictly sorted," and on the right the specific trip_id that contained the error is shown along with the stop_sequences that appeared out-of-order. If the user wants to look at this trip_id in more detail, they can scroll the left side of the screen or use a keyboard shortcut such as Ctrl-F for Find to locate this particular trip_id within the GTFS-realtime message. If a user wants to share this information with others, they can simply copy the URL for this iteration and send it to another person (e.g., via email). As an alternative, they can click on the "Download all” button to download all the error and warning occurrences for this iteration into a comma-separated value 
(CSV) file that can be opened in tools like Microsoft Excel (Figure 15). The user can also click on the download button for just a single error if they want to download only occurrences of that specific error.

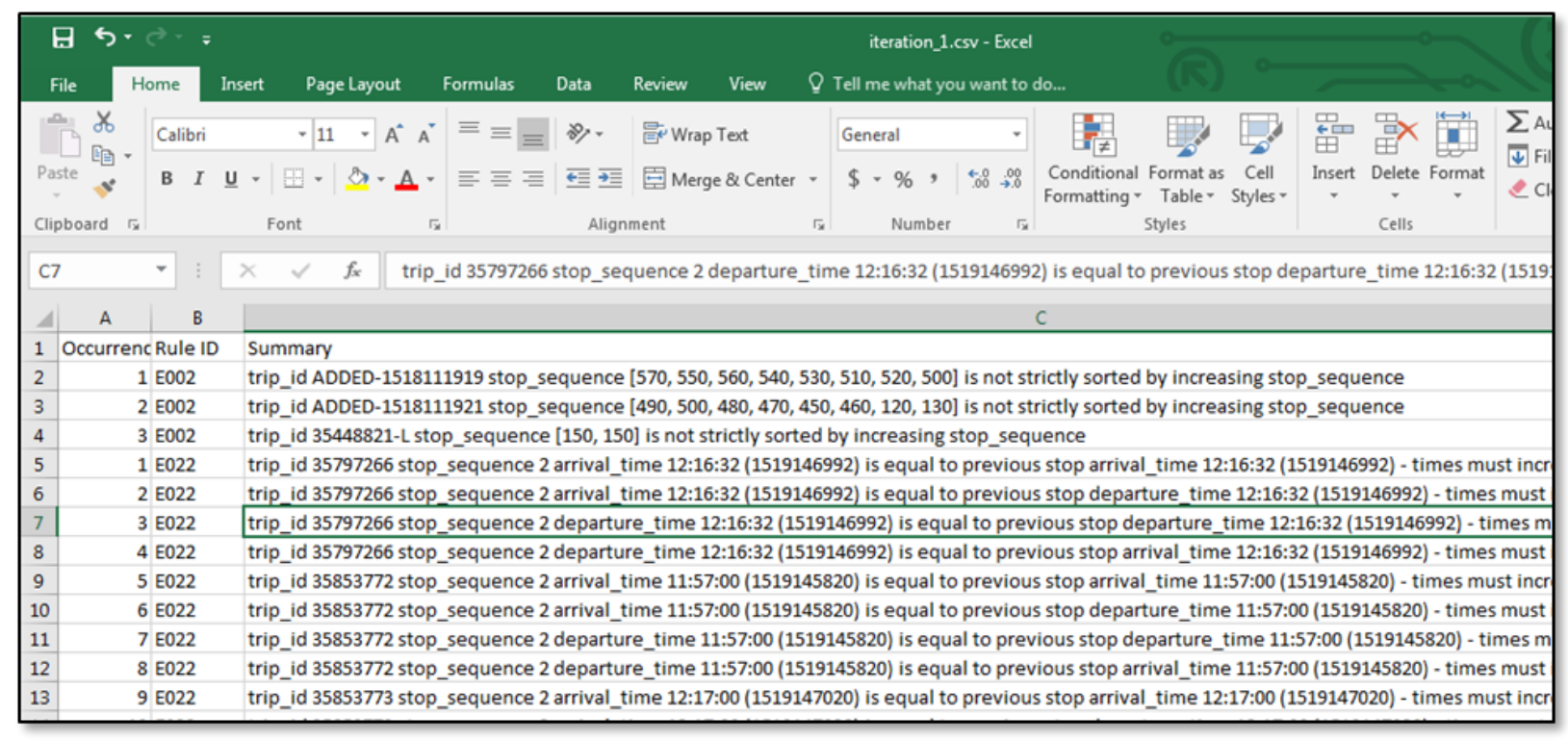

Figure 15 - All errors and warnings for a feed message can be downloaded in CSV format

\subsection{VALIDATION RULES}

The GTFS-realtime Validator has a modular rule architecture that allows new errors and warnings to be easily added to the tool as the GTFS-rt specification continues to evolve and new problems are discovered in feeds. As of February 2018, the research team has implemented rules to detect 47 types of errors and nine types of warnings [31], many of which were encountered in the team's experience analyzing real GTFS-realtime feeds. Tables of the currently implemented errors and warnings are shown in Table 4.1 and Table 4.2.

Table 4.1 - Errors currently implemented in the GTFS-realtime Validator

\begin{tabular}{|l|l|}
\hline Error ID & Error Title \\
\hline E001 & Not in POSIX time \\
\hline E002 & stop_time_updates not strictly sorted \\
\hline E003 & GTFS-rt trip_id does not exist in GTFS data \\
\hline E004 & GTFS-rt route_id does not exist in GTFS data \\
\hline E006 & Missing required trip field for frequency-based exact_times = 0 \\
\hline E009 & GTFS-rt stop_sequence isn't provided for trip that visits same stop_id more than once \\
\hline E010 & location_type not 0 in stops.txt \\
\hline E011 & GTFS-rt stop_id does not exist in GTFS data \\
\hline E012 & Header timestamp should be greater than or equal to all other timestamps \\
\hline E013 & Frequency type 0 trip schedule_relationship should be UNSCHEDULED or empty \\
\hline E015 & All stop_ids referenced in GTFS-rt feeds must have the location_type = 0 \\
\hline
\end{tabular}




\begin{tabular}{|c|c|}
\hline E016 & trip_ids with schedule_relationship ADDED must not be in GTFS data \\
\hline E017 & GTFS-rt content changed but has the same header timestamp \\
\hline E018 & GTFS-rt header timestamp decreased between two sequential iterations \\
\hline$\overline{\text { E019 }}$ & $\begin{array}{l}\text { GTFS-rt frequency type } 1 \text { trip start_time must be a multiple of GTFS headway_secs } \\
\text { later than GTFS start_time }\end{array}$ \\
\hline E020 & Invalid start_time format \\
\hline E021 & Invalid start_date format \\
\hline E022 & Sequential stop_time_update times are not increasing \\
\hline E023 & trip start_time does not match first GTFS arrival_time \\
\hline E024 & trip direction_id does not match GTFS data \\
\hline E025 & stop_time_update departure time is before arrival time \\
\hline E026 & Invalid vehicle position \\
\hline E027 & Invalid vehicle bearing \\
\hline E028 & Vehicle position outside agency coverage area \\
\hline E029 & Vehicle position far from trip shape \\
\hline E030 & GTFS-rt alert trip_id does not belong to GTFS-rt alert route_id in GTFS trips.txt \\
\hline E031 & Alert informed_entity.route_id does not match informed_entity.trip.route_id \\
\hline E032 & Alert does not have an informed_entity \\
\hline E033 & Alert informed_entity does not have any specifiers \\
\hline E034 & GTFS-rt agency_id does not exist in GTFS data \\
\hline E035 & GTFS-rt trip.trip_id does not belong to GTFS-rt trip.route_id in GTFS trips.txt \\
\hline E036 & Sequential stop_time_updates have the same stop_sequence \\
\hline E037 & Sequential stop_time_updates have the same stop_id \\
\hline E038 & Invalid header.gtfs_realtime_version \\
\hline E039 & FULL_DATASET feeds should not include entity.is_deleted \\
\hline E040 & stop_time_update doesn't contain stop_id or stop_sequence \\
\hline E041 & trip doesn't have any stop_time_updates \\
\hline E042 & arrival or departure provided for NO_DATA stop_time_update \\
\hline E043 & stop_time_update doesn't have arrival or departure \\
\hline E044 & stop_time_update arrival/departure doesn't have delay or time \\
\hline E045 & GTFS-rt stop_time_update stop_sequence and stop_id do not match GTFS \\
\hline E046 & GTFS-rt stop_time_update without time doesn't have arrival/departure time in GTFS \\
\hline E047 & VehiclePosition and TripUpdate ID pairing mismatch \\
\hline E048 & header timestamp not populated (GTFS-rt v2.0 and higher) \\
\hline E049 & header incrementality not populated (GTFS-rt v2.0 and higher) \\
\hline E050 & timestamp is in the future \\
\hline E051 & GTFS-rt stop_sequence not found in GTFS data \\
\hline
\end{tabular}

Table 4.2 - Warnings currently implemented in the GTFS-realtime Validator

\begin{tabular}{|l|l|}
\hline Warning ID & Warning Title \\
\hline W001 & timestamps not populated \\
\hline W002 & vehicle_id not populated \\
\hline W003 & ID in one feed missing from the other \\
\hline W004 & vehicle speed is unrealistic \\
\hline W005 & Missing vehicle_id in trip_update for frequency-based exact_times = 0 \\
\hline W006 & trip_update missing trip_id \\
\hline W007 & Refresh interval is more than 35 seconds \\
\hline W008 & Header timestamp is older than 65 seconds \\
\hline W009 & schedule_relationship not populated \\
\hline
\end{tabular}

An error is logged when data in the feed is incorrect and would result in a transit rider seeing bad or missing real-time information as a result. A warning is logged when a feed contains data that 
would negatively affect some GTFS-rt consuming applications but either cannot be confirmed to be incorrect with $100 \%$ certainty or the GTFS-rt specification does not clearly indicate that the data or behavior is incorrect. For example, a vehicle speed value over 26 meters per second, or 60 miles per hour, is suspicious for most intercity buses but may be valid for high-speed rail. In another example of a warning, developers expect a GTFS-realtime feed to refresh its contents frequently (every 30-60 seconds), but the GTFS-rt specification doesn't require a minimum update frequency.

Validation rules can be broken down into the following categories:

- Header - Checks if header fields (e.g., feed version) are populated correctly

- Timestamps - Checks integrity of feed timestamps (e.g., in POSIX format, age of feed, sequential arrival/departure times are in increasing order)

- Stop Time Updates - Checks the integrity of predictions provided for each trip (e.g., order by stop_sequence, missing field values, conflicts with GTFS stop_times.txt data)

- Stops - Checks that stop information provided in the feeds matches GTFS stops.txt (e.g., stop_id, location_type)

- Trip Descriptors - Checks integrity of trip properties (e.g., conflicts with GTFS data, missing data for certain use cases, trip start date formats)

- Vehicle - Checks integrity of vehicle properties (e.g., valid position/bearing formats, unrealistic speed values that may be unit conversion errors, proximity of real-time position to assigned GTFS trip)

- Cross Feed - If multiple feeds entity types exist (e.g., VehiclePositions and TripUpdates), checks if content in one set of entities matches the content in the other set of entities (e.g., that all trip_id and vehicle_id pairings are consistent)

- Frequency Type Zero Trips - Checks conditions specific to trips defined in frequencies.txt with exact_times $=0$ (i.e., true frequency/headway-based service)

- Frequency Type One Trips - Checks conditions specific to trips defined in frequencies.txt with exact_times $=1$ (i.e., scheduled service modeled using a specified headway interval)

A detailed description of all rules is documented on GitHub [31], and this GitHub page will continue to be updated as new rules are added to the validator. Detailed instructions for adding new rules are available on GitHub [32]. 


\subsection{GTFS-REALTIME BATCH PROCESSOR}

The rules implemented in the GTFS-realtime Validator can be used as a library in other software applications by referencing the gtfs-realtime-validation-lib project [33].

Within this library is the Batch Processor, which is a tool anyone can easily run from the command-line or from within another software application to process a GTFS and GTFSrealtime feed that has been downloaded to the computer. This application then outputs the validation results in JavaScript Object Notation (JSON) format (Table 4.3).

Table 4.3 - Example output from the Batch Processor validation tool

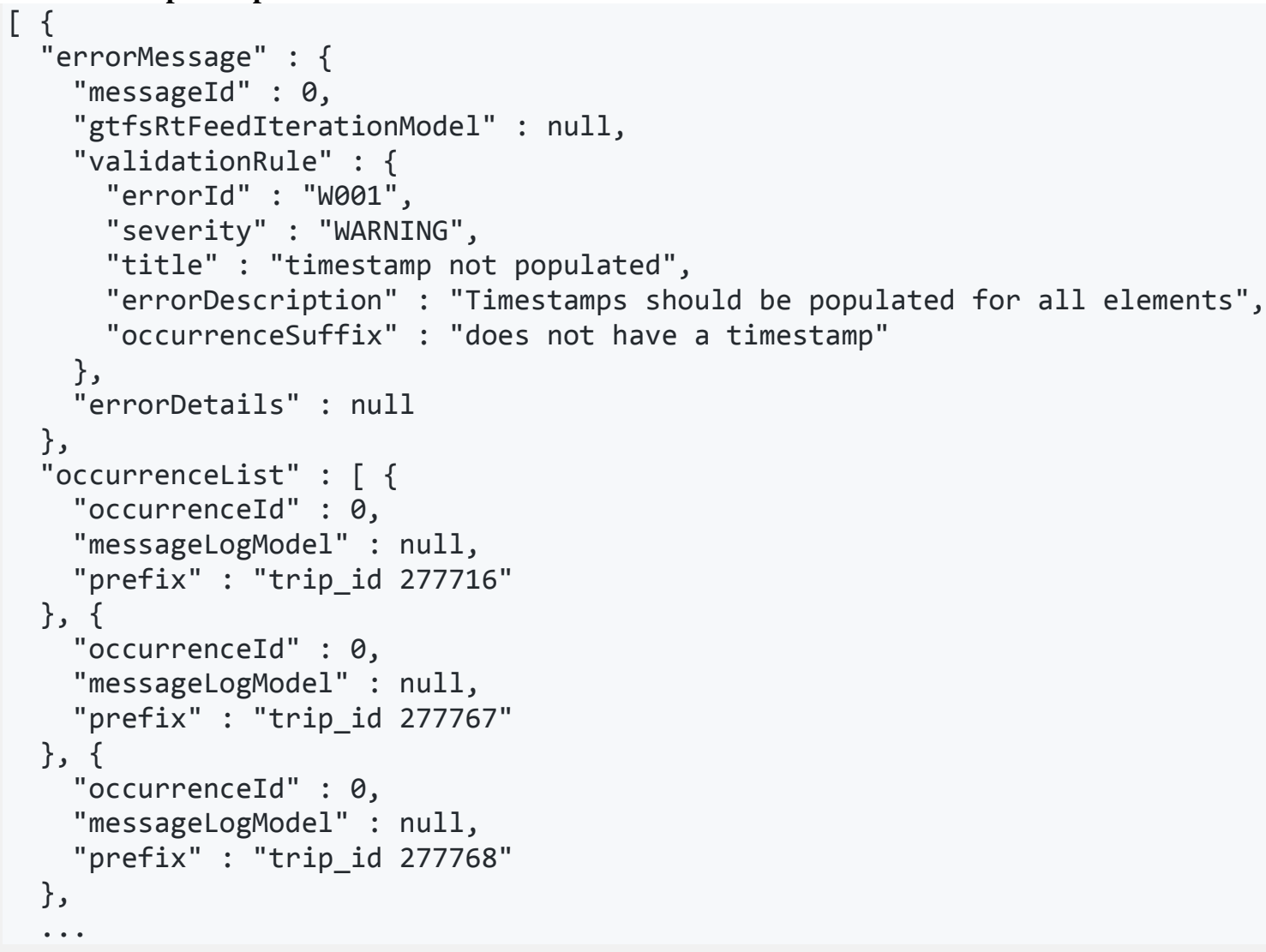

Detailed documentation for using the Batch Processor is available in the README of the gtfsrealtime-validation-lib project [33].

The following chapter showcases an example application that uses the Batch Processor to validate a large number of feeds downloaded from various transit agencies. 


\subsection{ANALYSIS OF GTFS-REALTIME FEEDS}

To demonstrate the industry need for the GTFS-realtime Validator, the research team developed another tool, the Transit Feed Quality Calculator [34], to automate the validation of a large number of live feeds from transit agencies.

This tool is easily executed from the command-line and:

- Retrieves the URLs for GTFS-realtime feeds and corresponding URLs for GTFS data from the TransitFeeds.com GetFeeds API (a centralized directory for GTFS and GTFSrealtime feed URLs)

- Downloads a snapshot of the GTFS-realtime and GTFS data from each agency's server into a subdirectory

- Runs the GTFS-realtime Validator on each of the subdirectories using the GTFS-realtime Validator Batch Processor library [33]

- Produces summary statistics and graphs for all validated feeds

While TransitFeeds.com shows a total of 130 GTFS-rt feeds that have been registered with the system [35], the team has so far been able to automate the validation of 78 feeds that are publicly available and do not have any access restrictions. As discussed later, future work will increase this number by supporting feeds that require application programming interface (API) keys or other types of authentication.

Out of the 78 feeds evaluated, 54 of the feeds contained errors and 58 of the feeds contained warnings (Figure 16). 


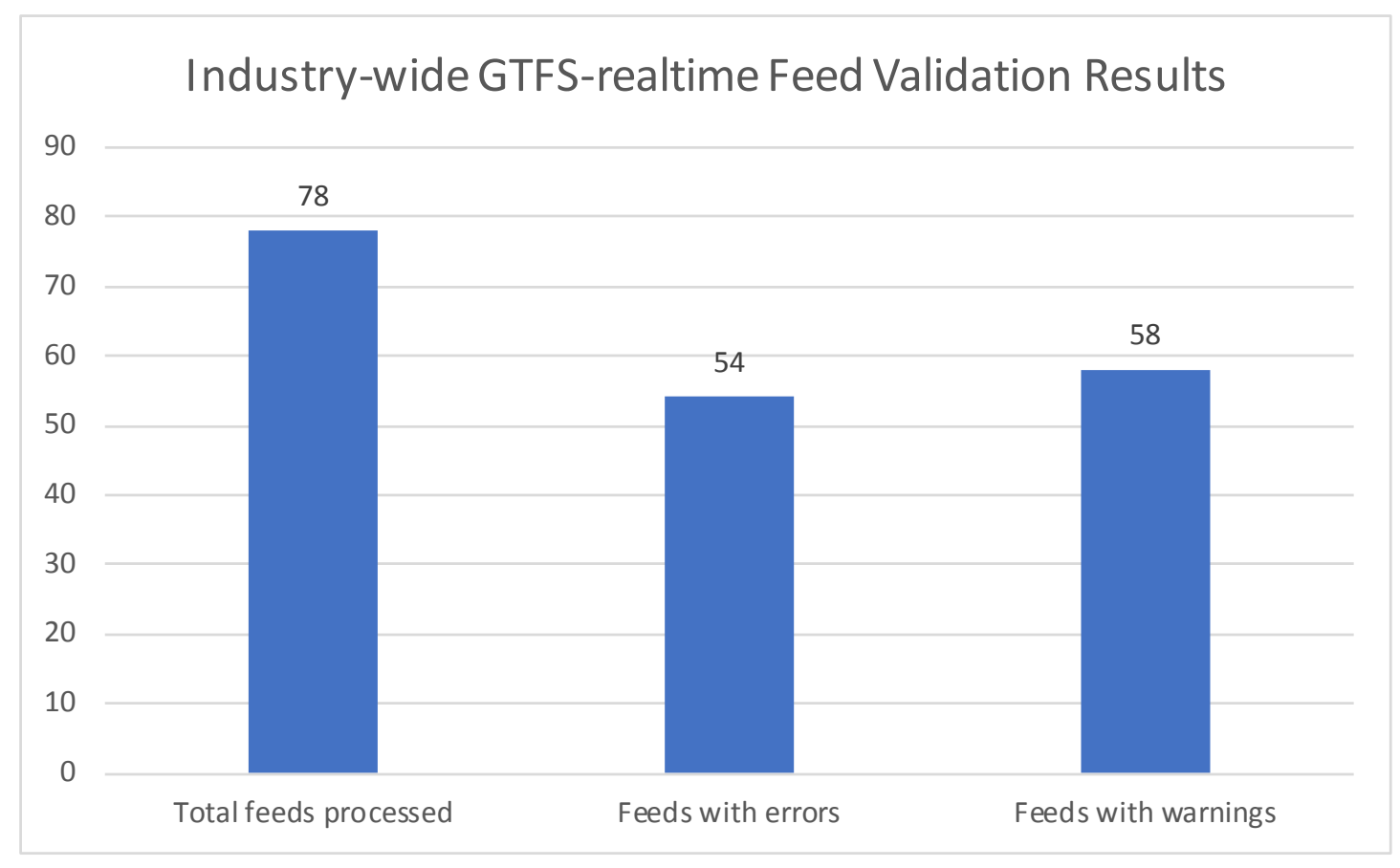

Figure 16 - Industry-wide GTFS-realtime Feed Validation Results

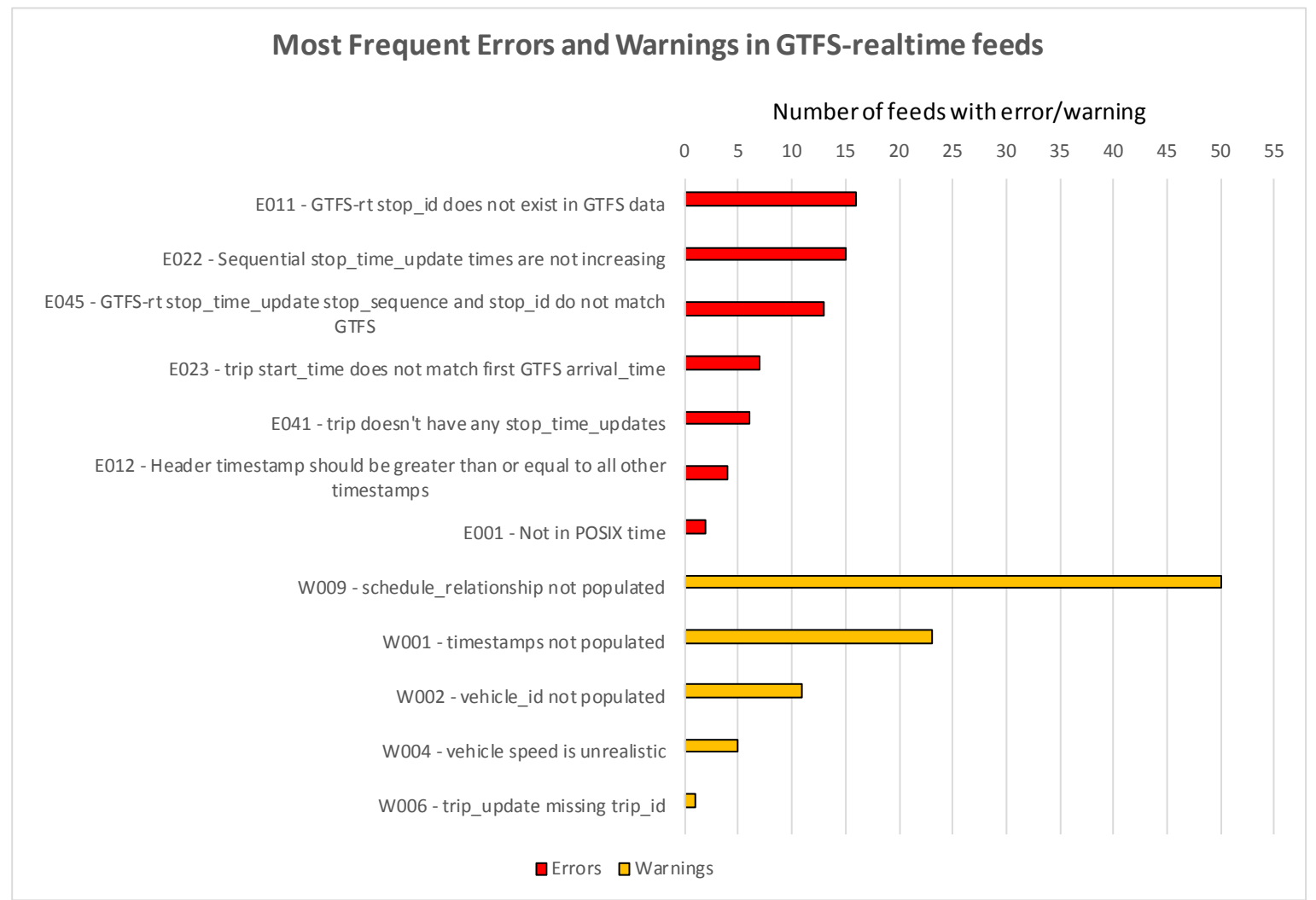

Figure 17 - Most Frequent Errors and Warnings in GTFS-realtime Feeds 
Figure 17 indicates the most common errors and warnings that appeared in feeds. "E011 GTFS-rt stop_id does not exist in GTFS data” was the most common error, appearing in 16 feeds. E011 occurs when the GTFS schedule data has no record of a stop that the GTFS-rt data is showing a prediction for, indicating an incorrect stop_id either in the GTFS or GTFS-rt data. The second most common error was "E022 - Sequential stop_time_update times are not increasing” appearing in 15 feeds (which indicates that predicted times are wrong - the vehicle would be traveling backwards in time). “E045 - GTFS-rt stop_time_update stop_sequence and stop_id do not match GTFS” appeared in 13 feeds - this means that the GTFS-rt data shows a conflicting order of arrival for stops for a trip when compared to the GTFS data.

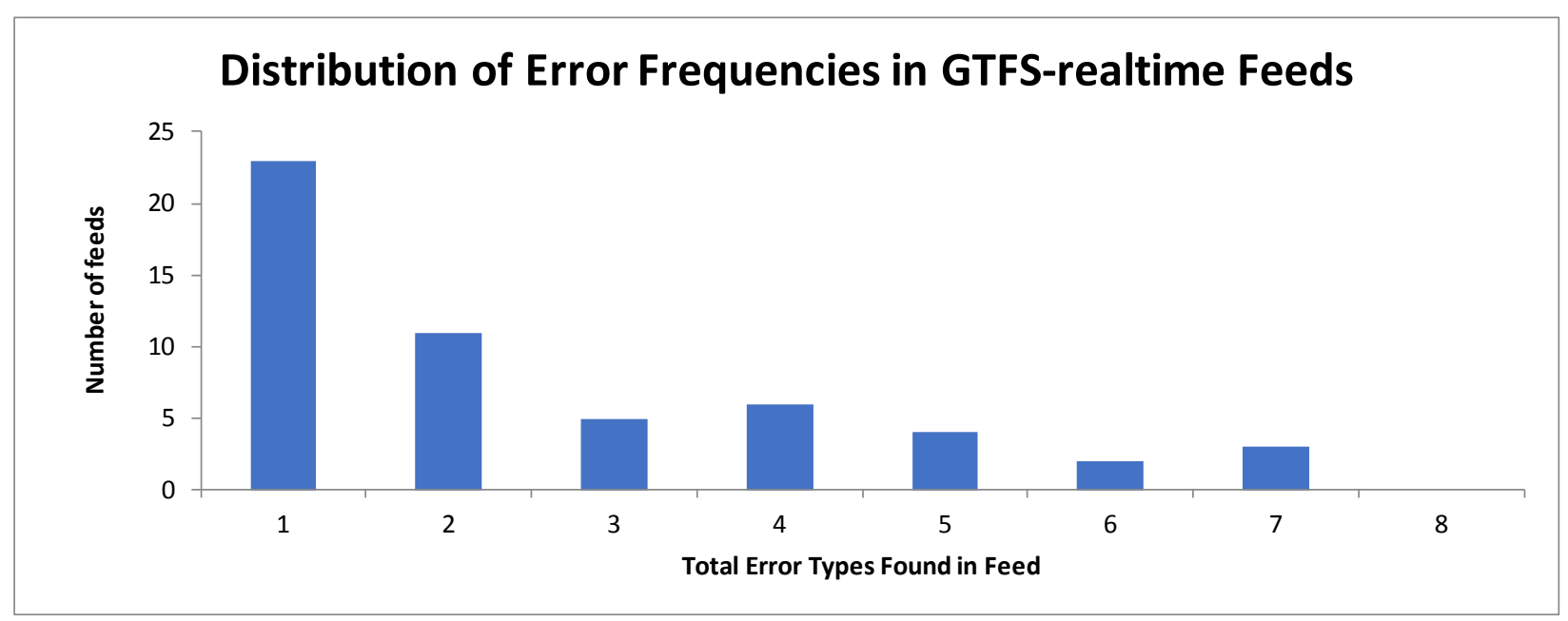

Figure 18 - Distribution of Error Frequencies in GTFS-realtime Feeds

Figure 18 shows the distribution of the count of error types found in feeds. For example, the feed with the worst performance had seven different types of errors, while 23 feeds had only one error type. Even though the majority of feeds had two or fewer types of errors, as mentioned earlier, some errors can occur multiple times in the same feed iteration, as well as in multiple iterations of the feed. For example, in a feed that had eight different types of errors, there were 24 occurrences of “E022 - Sequential stop_time_update times are not increasing” in a single feed iteration. Each of these occurrences can have a significant impact on the transit rider experience, as the rider would be viewing incorrect or missing arrival predictions (in the case that the consuming app filters out data that is obviously wrong).

The above analysis is for a single iteration of each of the 78 evaluated feeds. It is highly likely that if the validator was executed over multiple iterations of the feed (e.g., several hours of time) additional errors and warnings would be found for each feed. Some errors may originate from mis-configured schedule or real-time data that could potentially be fixed once and only occur again if the schedule data changes (e.g., when the agency releases a quarterly GTFS data update). 
Other errors may be harder to track down and could be related to bugs in the AVL vendor software or errors in vehicle operator logins that are less predictable in nature. As a result, it is recommended that agencies continuously monitor their real-time feeds so they are immediately aware of any problems that occur, especially when releasing a new GTFS dataset. 


\subsection{CONCLUSIONS AND FUTURE WORK}

The quality of real-time transit information is important to transit riders and transit agencies. As mentioned earlier, $9 \%$ of surveyed riders said that they took the bus less often due to errors they experienced. Prediction errors can also lead to reduced system performance if the operations department is making decisions based on this data.

This project has helped fill a vital gap in the state of the art of real-time transit information. The research team proposed GTFS-realtime v2.0, which defines fields as Required, Conditionally Required, and Optional based on real-world transit use cases. GTFS-realtime v2.0 was unanimously approved by the GTFS community in August 2017. The research team also contributed to the development of the GTFS Best Practices, as well as several other improvements to the GTFS and GTFS-realtime governance and specifications. An open-source GTFS-realtime Validator tool was developed, which transit agencies and AVL vendors can use to quickly identify problems in GTFS-realtime feeds. The research team also developed another tool, the Transit Feed Quality Calculator, which can quickly validate a large number of transit feeds using the GTFS-realtime Validator as a software library. This tool illustrates the challenges that the industry currently faces in the area of GTFS-realtime feed quality control, with 54 of the 78 evaluated feeds having a number of integrity errors in a single GTFS-realtime message. It is very likely that continued monitoring of these feeds over multiple messages would discover even more errors.

Going forward, the additional clarity in GTFS-realtime v2.0 and GTFS Best Practices should help feed producers provide better quality data, which should help drive this error count down. Additionally, the GTFS-realtime Validator should be easier for transit agencies and AVL vendors to identify problems in a feed so they can spend less time hunting for examples of errors and more time fixing the underlying problem.

\subsection{FUTURE WORK}

Now that GTFS-realtime v2.0 has been accepted by the community, future work should focus on encouraging transit agencies to adopt it, especially when they create Requests for Proposals (RFPs) for new AVL systems. The research team has created a blog post "What's new in GTFSrealtime v2.0" [14] that can be shared with interested parties, and has presented GTFS-realtime v2.0 at a TransitCenter Transit Data Workshop [12] as well as a paper and poster at the 2018 Transportation Research Board conference [13]. Future conference presentations and webinars can utilize these same materials. Given that the U.S. Department of Transportation has already requested GTFS data from agencies as part of the National Transit Map initiative [15], transit 
agencies may benefit from similar guidance regarding GTFS-realtime emerging as a de facto standard.

During the development of the GTFS-realtime Validator software, the research team discovered additional issues that require clarification via new proposals to the GTFS community, either by the research team or by others [16]. Future work should include collaborating with the GTFS community to write proposals to address these gray areas in the specification. Potential new rules (identified with the "new rule" label [17]) could also be added to the GTFS-realtime Validator, including a few that first require clarification in the GTFS-realtime specification. Areas requiring clarification are documented on GitHub [16] and include:

- Should stop_time_updates be propagated across trips in the same block?

- Are delays propagated downstream passed SKIPPED stops?

- Are stop_time_update.SKIPPED values propagated downstream?

- Should early arrivals/departures be propagated across timepoints?

- How to represent an alert that has no effect?

- What should consumer behavior for stop_time_updates with NO_DATA be?

- Do arrival and departure times need to be included in all stop_time_updates?

- Must stop_time_update stop and stop_sequence pairing match GTFS data stop_times.txt?

Several transit agencies voiced the desire to have a hosted instance of the GTFS-realtime Validator tool instead of running it themselves, largely due to internal agency IT restrictions that prevent the installation of new software on their computers. Future work should focus on establishing a long-term server where the GTFS-realtime Validator tool can be hosted - some preliminary work to achieve this goal has been accomplished and can be extended to support this task in the future [18].

Discussions with some transit agencies also uncovered a lack of knowledge for what is currently possible with GTFS-realtime. For example, some agencies were unaware that you could announce canceled service (e.g., trips) for a day and route in the feed. Future work could focus on creating better documentation in non-technical language that identifies these common use cases and clearly outlines what is and is not possible given the current specification.

Future work could also focus on creating official GTFS-realtime Best Practices documentation via the community process, similar to how the GTFS Best Practices were established. For example, many of the warnings created for the GTFS-realtime Validator are likely to be considered best practices by the majority of the community, and could be officially adopted via a vote.

The Transit Feed Quality Calculator tool can be improved. Future work could focus on compiling additional feeds not currently documented in TransitFeeds.com, as well as utilizing a 
CSV file to store some URLs for feeds that require API keys. Some preliminary work to collect data on which feeds require API keys has been done, and future work can build on these efforts [19].

Several agencies and application developers have expressed interest in a real-time "Data Dashboard" for GTFS-realtime feeds that would show the number of errors and warnings for all feeds for all agencies, as identified by the GTFS-realtime Validator. This website could be based on the hosted instance of the validator as discussed earlier or maintained as a separate service. Some have speculated that this would help personnel at all levels within the agency (from management to IT support) better understand how their agency is currently performing in the area of real-time data quality, and how they compare to their peers.

While the GTFS community has benefitted from the grassroots approach to governance in the past, given the substantial number of stakeholders for both GTFS and GTFS-realtime data the community may benefit from a more formal, organized structure going forward. The GTFS Best Practices effort showcases what can be accomplished relatively quickly by a smaller subset of organizations focused on accomplishing a specific goal, and a similar strategy will likely be required for creating GTFS-realtime Best Practices. This emerging coordination effort should be careful not to lose sight of the benefits of the grassroots approach that has made GTFS a widely adopted format (e.g., requiring producers and consumers for every new proposal to discourage speculative additions to the format). A more formal organization facilitating this coordination could also host useful resources and tools for producers and consumers of the data (e.g., the hosted GTFS-realtime Validator, the "Data Dashboard”).

Finally, there is research to be performed in the area of transit agency management and supporting information technology related to institutional barriers of acknowledging and resolving problems. For example, if a transit agency is provided with information to help them produce better quality transit data, will they take the steps to fix the problem? Would a hosted GTFS-realtime Validator or "Data Dashboard” help an agency improve their systems? The GTFS-realtime Validator tool can help produce quantitative metrics that could be tracked over time to try to answer some of these questions. 


\subsection{REFERENCES}

[1] Kari Edison Watkins, Brian Ferris, Alan Borning, G. Scott Rutherford, and David Layton (2011), "Where Is My Bus? Impact of mobile real-time information on the perceived and actual wait time of transit riders," Transportation Research Part A: Policy and Practice, Vol. 45 pp. 839-848.

[2] C. Cluett, S. Bregman, and J. Richman (2003). "Customer Preferences for Transit ATIS," Federal Transit Administration.

[3] Brian Ferris, Kari Watkins, and Alan Borning, "OneBusAway: results from providing real-time arrival information for public transit," presented at the Proceedings of the 28th international conference on Human factors in computing systems, Atlanta, Georgia, USA, 2010.

[4] A. Gooze, K. Watkins, and A. Borning (2013), "Benefits of Real-Time Information and the Impacts of Data Accuracy on the Rider Experience," in Transportation Research Board 92nd Annual Meeting, Washington, D.C., January 13, 2013.

[5] Lei Tang and Piyushimita Thakuriah (2012), "Ridership effects of real-time bus information system: A case study in the City of Chicago," Transportation Research Part C: Emerging Technologies, Vol. 22 pp. 146-161.

[6] C. Brakewood, G. Macfarlane, and K. Watkins (2015), "The impact of real-time information on bus ridership in New York City," Transportation Research Part C: Emerging Technologies, Vol. 53 pp. 59-75.

[7] C. Brakewood, S. Barbeau, and K. Watkins (2014), "An experiment evaluating the impacts of real-time transit information on bus riders in Tampa, Florida," Transportation Research Part A: Policy and Practice, Vol. 69 pp. 409-422.

[8] Google, Inc. "General Transit Feed Specification Reference." Accessed July 31, 2017 from https:/github.com/google/transit/blob/master/gtfs/spec/en/reference.md

[9] MapZen. "TransitLand - An Open Project - For Data Providers." Accessed July 31, 2017 from https://transit.land/an-open-project/

[10] Google, Inc. "GTFS Realtime Reference." Accessed August 1, 2017 from https://github.com/google/transit/blob/master/gtfs-realtime/spec/en/reference.md

[11] GTFS Best Practices Working Group. "GTFS Best Practices Working Group." Accessed February 28, 2018 from http://gtfs.org/best-practices/\#working-group

[12] S. Barbeau (2017), "GTFS-realtime v2.0," in TransitCenter Transit Data Workshop, New York, New York, October 18, 2017.

[13] Sean J. Barbeau (2018), "Quality Control - Lessons Learned from the Deployment and Evaluation of GTFS-realtime Feeds," in Transportation Research Board 97th Annual Meeting, Washington, D.C., January 7-11, 2018.

[14] Sean J. Barbeau, "What's new in GTFS-realtime v2.0," Vol. 2017, ed. Medium, 2017.

[15] U.S. Department of Transportation. "National Transit Map." Accessed February 28, 2018 from http://gtfs.org/best-practices/\#working-group

[16] University of South Florida. "GTFS-realtime - Issues requiring specification clarification." Accessed February 20, 2018 from https://github.com/CUTR-at-USF/gtfs- 
realtime-validator/issues?q=is\%3Aissue+is\%3Aopen+label\%3A\%22GTFS\%28rt\%29+spec+clarification\%22

[17] University of South Florida. "GTFS-realtime - Issues that could result in new validation rules." Accessed February 20, 2018 from https://github.com/CUTR-at-USF/gtfs-realtimevalidator/issues?q=is\%3Aissue+is\%3Aopen+label\%3A\%22new+rule\%22

[18] University of South Florida. "gtfs-realtime-validator - Set up hosted instance using CUTR server." Accessed February 20, 2018 from https://github.com/CUTR-at-USF/gtfsrealtime-validator/issues/308

[19] University of South Florida. "transit-feed-quality-calculator - Gather API keys for transit feeds that require them." Accessed February 20, 2018 from https://github.com/CUTR-atUSF/transit-feed-quality-calculator/issues/29

[20] S. Barbeau (2013), "Open Transit Data - A Developer’s Perspective," in APTA TransITech 2013, Phoenix, Arizona, March 20th, 2013.

[21] Google, Inc. "Protocol Buffers." Accessed July 31, 2017 from https://developers.google.com/protocol-buffers/

[22] Google, Inc. "gtfs-realtime-bindings." Accessed February 14, 2018 from https://github.com/google/gtfs-realtime-bindings

[23] Google, Inc. "Protocol Buffers - Specifying Field Rules - Required is Forever." Accessed July 31, 2017 from https://developers.google.com/protocolbuffers/docs/proto\#specifying-field-rules

[24] GTFS-realtime Google Group. "Proposal: Make FeedHeader.timestamp a required field." Accessed January 2015 from https://groups.google.com/forum/\#!msg/gtfsrealtime/wm3W7QIEZ9Y/DLyWKkknJyoJ

[25] Sean J. Barbeau. "Pull Request \#64 - Define semantic cardinality for GTFS-realtime fields." Accessed November 14, 2017 from https://github.com/google/transit/pull/64

[26] GTFS Community. "GTFS Data Best Practices." Accessed February 14, 2018 from http://gtfs.org/best-practices/

[27] Google, Inc. "Merge." Accessed February 14, 2018 from https://github.com/google/transitfeed/wiki/Merge

[28] University of South Florida. "GTFS Validators." Accessed February 20, 2018 from https://github.com/CUTR-at-USF/awesome-transit\#gtfs-validators

[29] Massashusetts Bay Transportation Authority. "Developer Portal." Accessed November 14, 2017 from http://realtime.mbta.com/Portal/Home/Documents

[30] University of South Florida. "GTFS-realtime Validator." Accessed November 14, 2017 from https://github.com/CUTR-at-USF/gtfs-realtime-validator

[31] University of South Florida. "GTFS-realtime Validator - Implemented Rules." Accessed February 20, 2018 from https://github.com/CUTR-at-USF/gtfs-realtimevalidator/blob/master/RULES.md

[32] University of South Florida. "GTFS-realtime Validator - Adding New Rules." Accessed February 20, 2018 from https://github.com/CUTR-at-USF/gtfs-realtimevalidator/blob/master/ADDING_NEW_RULES.md

[33] University of South Florida. "GTFS-realtime Validator - Batch Processor." Accessed November 14, 2017 from https://github.com/CUTR-at-USF/gtfs-realtimevalidator/tree/master/gtfs-realtime-validator-lib

[34] University of South Florida. "transit-feed-quality-calculator." Accessed November 15, 2017 from https://github.com/CUTR-at-USF/transit-feed-quality-calculator 
[35] TransitFeeds.com. "TransitFeeds.com." Accessed November 10, 2016 from http://transitfeeds.com/ 


\section{Transportation Research and Education Center}

Portland State University

1900 S.W. Fourth Ave., Suite 175

Portland, OR 97201 